\title{
MINIMAL SURFACES IN SUB-RIEMANNIAN MANIFOLDS AND STRUCTURE OF THEIR SINGULAR SETS IN THE $(2,3)$ CASE
}

\author{
NATALIYA SHCHERBAKOVA ${ }^{1}$
}

\begin{abstract}
We study minimal surfaces in sub-Riemannian manifolds with sub-Riemannian structures of co-rank one. These surfaces can be defined as the critical points of the so-called horizontal area functional associated with the canonical horizontal area form. We derive the intrinsic equation in the general case and then consider in greater detail 2-dimensional surfaces in contact manifolds of dimension 3. We show that in this case minimal surfaces are projections of a special class of 2-dimensional surfaces in the horizontal spherical bundle over the base manifold. The singularities of minimal surfaces turn out to be the singularities of this projection, and we give a complete local classification of them. We illustrate our results by examples in the Heisenberg group and the group of roto-translations.
\end{abstract}

Mathematics Subject Classification. 53C17, 32S25.

Received August 29, 2007. Revised April 20, 2008.

Published online July 19, 2008.

\section{INTRODUCTION}

In the classical Riemannian geometry minimal surfaces realize the critical points of the area functional with respect to variations preserving the boundary of a given domain. In this paper we study the generalization of the notion of minimal surfaces in sub-Riemannian manifolds known also as the Carnot-Carathéodory spaces. This problem was first introduced in the framework of Geometric Measure Theory for the Lie groups. Mainly the obtained results $[6,7,10-12,15,16]$ concern the Heisenberg groups, in particular $\mathbb{H}^{1}$; in $[9,13]$ the authors were studying the group $E_{2}$ of roto-translations of the plane, in [7] there were also obtained some results for the case of $S^{3}$. In [7], followed by just appeared paper [8], the authors considered the problem in a more general setting and introduced the notion of minimal surfaces associated with CR structures in pseudohermitian manifolds of any dimension.

In this paper we develop a different approach using the methods of sub-Riemannian geometry. Though in particular cases of Lie groups $\mathbb{H}^{m}, E_{2}$ and $S^{3}$ the surfaces introduced in [7] are minimal also in the subRiemannian sense, in general it is not true. The sub-Riemannian point of view on the problem is based on the following construction.

\footnotetext{
Keywords and phrases. Sub-Riemannian geometry, minimal surfaces, singular sets.
}

1 SISSA/ISAS, via Beirut 2-4, 34100, Trieste, Italy. chtch@sissa.it 
Consider an $n$-dimensional smooth manifold $M$ and a co-rank 1 smooth vector distribution $\Delta$ in it ("horizontal" distribution). It is assumed that the sections of $\Delta$ are endowed with a Euclidean structure, which can be described by fixing an orthonormal basis of vector fields $X_{1}, \ldots, X_{n-1}$ on $\Delta$ (see [5]). Then $\Delta$ defines a $s u b$ Riemannian structure in $M$. In this case $M$ is said to be a sub-Riemannian manifold. Given a sub-Riemannian structure there is a canonical way to define a volume form $\mu \in \Lambda^{n} M$ associated with it. In addition, for any hypersurface $W \subset M$ the horizontal unite vector $\nu$ such that for any bounded domain $\Omega \subset W$

$$
\int_{\Omega} i_{\nu} \mu=\sup _{\substack{X \in \Delta \\\|X\| \Delta}} \int_{\Omega} i_{X} \mu,
$$

plays the role of the Riemannian normal in the classical case, and the $n-1$-form $i_{\nu} \mu$ defines the horizontal area form on $W$. All these notions are direct generalizations of the classical ones in the Riemannian geometry (i.e., in the case $\Delta \equiv T M)$.

Going further in this direction, we define sub-Riemannian minimal surfaces in $M$ as the critical points of the functional associated with the horizontal area form. It turns out that these surfaces satisfy the following intrinsic equation

$$
\left.\left(d \circ i_{\nu} \mu\right)\right|_{W \backslash \Sigma}=0,
$$

where $\Sigma=\left\{q \in W \mid T_{q} W \subseteq \Delta_{q}\right\}$ is the singular set of $W$, which along with the singular points of $W$ contains also the so-called characteristic points, i.e., the points where $W$ is tangent to $\Delta$. The described construction does not require the existence of any additional global structure in $M$, and can be generalized for sub-Riemannian structures of greater co-rank.

The existence of the singular set $\Sigma$ is one of the main difficulties of the problem. In general, the set $\Sigma$ can be quite large and have its own non-trivial intrinsic geometry. In Section 2 of this paper we show how this problem can be resolved in the case of 2-dimensional surfaces in 3-dimensional contact manifolds.

It turns out that in the $(2,3)$ case, due to the relatively small dimension, there is an elegant way to extend the definition of a sub-Riemannian minimal surface over its singular set. Namely, in this case the intrinsic geometry of a surface $W$ is encoded in its characteristic curves $\gamma:[0, T] \rightarrow W$ such that $\dot{\gamma}(t) \in T_{\gamma(t)} W \cap \Delta_{\gamma(t)}$ for all $t \in[0, T]$. The vector field $\eta$ (the characteristic vector field) tangent to characteristic curves is $\Delta$-orthogonal to the sub-Riemannian normal of $W$. We show that actually this vector field is a projection onto $M$ of a special invariant vector field $V$ in the horizontal spherical bundle $S_{\Delta} M$ over $M$. In contrast with $\nu$, the vector field $V$ is well defined everywhere in $S_{\Delta} M$, and moreover, minimal surface equation (0.1) can be transformed into a quasilinear equation whose characteristics are exactly the integral curves of $V$. In particular, in this way one can define $\eta$ also on $\Sigma$ as the projection of $V$.

These observations motivated the key idea of the present paper. By introducing an additional scalar parameter $\varphi$ we show that the highly degenerate PDE (0.1) can be transformed into a system of ODEs on $S_{\Delta} M$, associated with the vector field $V: \dot{\bar{q}}=V(\bar{q}), \bar{q} \in S_{\Delta} M$. This allows us to consider the sub-Riemannian minimal surfaces in the $(2,3)$ case as the projections of a certain class of 2-dimensional surfaces in $S_{\Delta} M$ foliated by the integral curves of $V$ (the generating surfaces of the sub-Riemannian minimal surfaces). By varying initial conditions, one can provide a local characterization of all possible sub-Riemannian minimal surfaces, together with their singular sets.

From the point of view that we develop in this paper the set $\Sigma$ may contain

- projections of the singular points of the generating surfaces;

- singularities of the projection of the generating surface onto the base manifold $M$ (singular characteristic points);

- regular points of the projection of the generating surface onto $M$ (regular characteristic points).

In this work we focus out attention on the case of regular generating surfaces. Then their projections on $M$ can have characteristic points of the last two types. We show that regular characteristic points form simple 
singular curves. Moreover, generically a small neighborhood of a sub-Riemannian minimal surface containing a singular characteristic point $q$ has a structure of Whitney's umbrella, and the point $q$ gives rise to a curve of self-intersections and a pair of simple singular curves (see Thm. 2.9 in Sect. 2). Other types of singularities may appear in some particular cases. For instance, certain minimal surfaces may contain isolated characteristic points or entire curves of singular points (strongly singular curves).

All described types of singularities are already present in the Heisenberg group $\mathbb{H}^{1}$. In Section 3 we exhibit several examples to illustrate our results.

There are still many natural questions concerning the structure of sub-Riemannian minimal surfaces, which are not touched in the present paper. First of all, we consider only surfaces generated by smooth curves in $S_{\Delta} M$. Then, we do not discuss the problem of gluing together smooth surfaces, as well as the existence of a smooth minimizing surface spanning a given contour. We leave these problems as the topics for the further studies.

\section{Minimal SURFACES in SUb-Riemannian MANifoldS}

\subsection{Sub-Riemannian structures and associated objects}

Let $M$ be an $n$-dimensional smooth manifold. Consider a co-rank 1 vector distribution $\Delta$ in $M$ :

$$
\Delta=\bigcup_{q \in M} \Delta_{q}, \quad \Delta_{q} \subset T_{q} M, \quad q \in M .
$$

By definition, a sub-Riemannian structure in $M$ is a pair $\left(\Delta,\langle\cdot, \cdot\rangle_{\Delta}\right)$, where $\langle\cdot, \cdot\rangle_{\Delta}$ denotes a smooth family of Euclidean inner products on $\Delta$. In what follows we will call $\Delta$ the horizontal distribution and keep the same notation $\Delta$ both for the vector distribution and for the associated sub-Riemannian structure.

Let $X_{i}, i=1, \ldots, n-1$, be a horizontal orthonormal basis:

$$
\begin{gathered}
\Delta_{q}=\operatorname{span}\left\{X_{1}(q), \ldots, X_{n-1}(q)\right\}, \quad q \in M, \\
\left\langle X_{i}(q), X_{j}(q)\right\rangle_{\Delta}=\delta_{i j}, \quad q \in M, i, j=1, \ldots, n-1 .
\end{gathered}
$$

By $\Theta \in \Lambda^{n-1} \Delta$ we will denote the Euclidean volume form on $\Delta$. Throughout this paper we assume that the fields $X_{i}, i=1, \ldots, n-1$, are defined everywhere on $M$.

In what follows we will also assume that $\Delta$ is bracket-generating in the sense that

$$
\operatorname{span}\left\{X_{i}(q),\left[X_{i}, X_{j}\right](q), i, j=1, \ldots, n-1\right\}=T_{q} M, \quad q \in M .
$$

Hereafter the square brackets denote the Lie brackets of vector fields. If $\Delta$ is bracket-generating, then by the Frobenius theorem it is completely non-holonomic, i.e., there is no invariant sub-manifold in $M$ whose tangent space coincides with $\Delta$ at any point.

We can also define the distribution $\Delta$ as the kernel of some differential 1-form. Let $\omega \in \Lambda^{1} M$ be such a form:

$$
\Delta_{q}=\operatorname{Ker} \omega_{q}=\left\{v \in T_{q} M \mid \omega_{q}(v)=0\right\}, \quad q \in M .
$$

It is easy to check that $\Delta$ is bracket-generating at $q \in M$ if and only if $d_{q} \omega \neq 0$.

Though in general the form $\omega$ is defined up to a multiplication by a non-zero scalar function, there is a canonical way to choose it by using the Euclidean structure on $\Delta$. Indeed, by standard construction the Euclidean structure on $\Delta$ can be extended to the spaces of forms $\Lambda^{k} \Delta, k \leq n-1$. In particular, for any 2 -form $\sigma$ we set

$$
\left\|\sigma_{q}\right\|_{\Delta}=\left(\sum_{\substack{i, j=1 \\ i<j}}^{n-1} \sigma_{q}\left(X_{i}(q), X_{j}(q)\right)^{2}\right)^{\frac{1}{2}},
$$


$\left\{X_{i}(q)\right\}_{i=1}^{n-1}$, as before, being the orthonormal horizontal basis of $\Delta_{q}$. Now we can normalize $\omega$ as follows:

$$
\omega_{q}\left(\Delta_{q}\right)=0, \quad\left\|\left.d_{q} \omega\right|_{\Delta}\right\|_{\Delta}^{2}=1, \quad \forall q \in M .
$$

In the fixed horizontal orthonormal basis $\left\{X_{i}(q)\right\}_{i=1}^{n-1} \in \Delta_{q}$ equations (1.1) become

$$
\omega_{q}\left(X_{i}(q)\right)=0, \quad \sum_{\substack{i, j=1 \\ i<j}}^{n-1} d_{q} \omega\left(X_{i}(q), X_{j}(q)\right)^{2}=1, \quad i=1, \ldots, n-1 .
$$

Clearly the 1-form $\omega$ is defined by equations (1.2) up to a sign and it is invariant with respect to the choice of the horizontal basis. In what follows we will call $\omega$ satisfying (1.1) the canonical 1-form associated with the sub-Riemannian structure $\Delta$. In local coordinates on $M$ the components of the canonical form $\omega$ can be expressed in terms of the coordinates of the vector fields $X_{i}$ and their first derivatives. Indeed, we have

$$
\left\|\left.d_{q} \omega\right|_{\Delta}\right\|_{\Delta}^{2}=\sum_{\substack{i, j=1 \\ i<j}}^{n-1} d_{q} \omega\left(X_{i}(q), X_{j}(q)\right)^{2}=\sum_{\substack{i, j=1 \\ i<j}}^{n-1} \omega_{q}\left(\left[X_{i}, X_{j}\right](q)\right)^{2}
$$

because according to Cartan's formula for any pair of vector fields $X$ and $Y$

$$
d \omega(X, Y)=X \omega(Y)-Y \omega(X)-\omega([X, Y]) .
$$

Once the orientation in $M$ is fixed by a choice of the sign of $\omega$, the volume form

$$
\mu=\Theta \wedge \omega
$$

is uniquely defined. We will call this volume form the canonical volume form associated with $\Delta$.

\subsection{Horizontal area form}

Let $W \subset M, \operatorname{dim} W=n-1$, be a smooth hypersurface in $M$ and let $\Omega$ be a bounded domain in $W$. Let $X \in \operatorname{Vec}(M)$ be a smooth vector field and denote by $\mathrm{e}^{t X}$ the flow generated by $X$ in $M$. Consider the map

$$
\begin{gathered}
\Pi^{X}: \quad[0, \varepsilon] \times \Omega \rightarrow M, \\
\Pi^{X}(t, q)=\mathrm{e}^{t X}(q), \quad q \in M .
\end{gathered}
$$

Let us denote by

$$
\Pi_{(\varepsilon, \Omega)}^{X}=\left\{\mathrm{e}^{t X}(q) \mid q \in \Omega, t \in[0, \varepsilon]\right\}
$$

the cylinder formed by the images of $\Omega$ translated along the integral curves of $X$ parameterized by $t \in[0, \varepsilon]$. Clearly, $\Pi_{(0, \Omega)}^{X}=\Omega$. By definition,

$$
\operatorname{Vol}\left(\Pi_{(\varepsilon, \Omega)}^{X}\right)=\int_{\Pi_{(\varepsilon, \Omega)}^{X}} \mu=\int_{[0, \varepsilon] \times \Omega}\left(\Pi^{X}\right)^{*} \mu,
$$

where $\left(\Pi^{X}\right)^{*}$ is the pull-back map associated with $\Pi^{X}$, and $\mu$ is the canonical volume form defined above ${ }^{1}$.

Before going further observe that since $\left(\Pi^{X}\right)^{*} \mu$ is a form of maximal rank $n$ in $M$ we have $d t \wedge\left(\Pi^{X}\right)^{*} \mu=0$. Hence

$$
0=i_{\partial_{t}}\left(d t \wedge\left(\Pi^{X}\right)^{*} \mu\right)=i_{\partial_{t}} d t \wedge\left(\Pi^{X}\right)^{*} \mu-d t \wedge i_{\partial_{t}}\left(\Pi^{X}\right)^{*} \mu,
$$

\footnotetext{
${ }^{1}$ Here we use the canonical volume form associated with $\Delta$, though the whole construction works for any volume form in $M$.
} 
i.e.,

$$
\left(\Pi^{X}\right)^{*} \mu=d t \wedge i_{\partial_{t}}\left(\Pi^{X}\right)^{*} \mu .
$$

Taking into account that $\Pi_{*}^{X} \partial_{t}=X$ we obtain

$$
\operatorname{Vol}\left(\Pi_{(\varepsilon, \Omega)}^{X}\right)=\int_{[0, \varepsilon] \times \Omega}\left(\Pi^{X}\right)^{*} \mu=\int_{[0, \varepsilon] \times \Omega} d t \wedge i_{\partial_{t}}\left(\Pi^{X}\right)^{*} \mu=\int_{0}^{\varepsilon}\left(\int_{\Pi_{(t, \Omega)}^{X}} i_{X} \mu\right) \mathrm{d} t .
$$

In particular, it follows that

$$
\lim _{\varepsilon \rightarrow 0} \frac{\operatorname{Vol}\left(\Pi_{(\varepsilon, \Omega)}^{X}\right)}{\varepsilon}=\left.\frac{\mathrm{d}}{\mathrm{d} \varepsilon}\right|_{\varepsilon=0} \operatorname{Vol}\left(\Pi_{(\varepsilon, \Omega)}^{X}\right)=\int_{\Omega} i_{X} \mu .
$$

Definition 1.1. We will call

$$
A_{\Delta}(\Omega)=\sup _{\substack{X \in \Delta \\\|X\|_{\Delta}=1}} \int_{\Omega} i_{X} \mu
$$

the sub-Riemannian (or horizontal) area of the domain $\Omega$ associated with $\Delta$.

Remark 1.2. The horizontal area (1.4) is a natural generalization of the classical notion of the Euclidean area: it defines the area of the base of a cylinder as the ratio of its volume and height.

Definition 1.3. The horizontal unit vector field $\nu \in \Delta,\|\nu\|_{\Delta}=1$, such that for any bounded domain $\Omega \subset W$

$$
\int_{\Omega} i_{\nu} \mu=\sup _{\substack{X \in \Delta \\\|X\|_{\Delta}=1}} \int_{\Omega} i_{X} \mu
$$

is called the sub-Riemannian or horizontal normal of $W$. The $(n-1)$-form $i_{\nu} \mu$ is called the sub-Riemannian or horizontal area form on $W$ associated with $\Delta$.

Remark 1.4. According to the definition, the horizontal normal $\nu$ is well defined everywhere except the points where the surface $W$ is tangent to the distribution $\Delta$. Such points are called the characteristic points of $W$ and they belong to the subset

$$
\Sigma=\left\{q \in W \mid T_{q} W \subseteq \Delta_{q}\right\}
$$

called the singular set of $W$. The set $\Sigma$ can have a very non-trivial intrinsic geometry. In Section 2 we will analyze in detail the structure of $\Sigma$ in the case $\operatorname{dim} M=3$.

The sub-Riemannian normal is an intrinsic object associated with any hypersurface in $M$, and Definition 1.3 does not require any other global structure in $M$ besides $\Delta$. Nevertheless, if $M$ is endowed with a Riemannian structure compatible with the sub-Riemannian structure on $\Delta$, i.e., the inner product $\langle\cdot, \cdot\rangle$ on $T M$ satisfies $\langle\cdot, \cdot\rangle_{\Delta}=\left.\langle\cdot, \cdot\rangle\right|_{\Delta}$, then it is easy to see that the sub-Riemannian normal $\nu$ is nothing but the projection on $\Delta$ of the Riemannian unit normal $N$ of $W$, normalized w.r.t. $\|\cdot\|_{\Delta}$. This fact follows from the relation

$$
\int_{\Omega} i_{X} \mu=\int_{\Omega}\langle X, N\rangle i_{N} \mu, \quad \forall X \in \operatorname{Vec}(M) .
$$

Thus if $X_{1}, \ldots, X_{n-1} \in \Delta$ is an orthonormal horizontal basis of $\Delta$, then

$$
\nu=\sum_{i=1}^{n-1} \nu_{i} X_{i}, \quad \nu_{i}=\frac{\left\langle N, X_{i}\right\rangle}{\sqrt{\left\langle N, X_{1}\right\rangle^{2}+\ldots+\left\langle N, X_{n-1}\right\rangle^{2}}},
$$


and the horizontal area form reads

$$
i_{\nu} \mu=\langle\nu, N\rangle i_{N} \mu=\sqrt{\left\langle N, X_{1}\right\rangle^{2}+\ldots+\left\langle N, X_{n-1}\right\rangle^{2}} i_{N} \mu .
$$

Consider now a hypersurface $W$ defined as a zero level set of a smooth, let us say $C^{2}$, function:

$$
W=\left\{q \in M \mid F(q)=0, d_{q} F \neq 0\right\}, \quad F \in C^{2}(M) .
$$

If $X \equiv X_{n} \in \operatorname{Vec}(M)$ is such that $\left\{X_{i}(q)\right\}_{i=1}^{n}$ is an orthonormal basis of $T_{q} M$ at $q \in M$, then

$$
N(q)=D_{0}^{-1} \sum_{i=1}^{n} X_{i} F(q) X_{i}(q), \quad D_{0}=\left(\sum_{i=1}^{n} X_{i} F(q)^{2}\right)^{1 / 2}
$$

and

$$
\nu(q)=D_{1}^{-1} \sum_{i=1}^{n-1} X_{i} F(q) X_{i}(q), \quad D_{1}=\left(\sum_{i=1}^{n-1} X_{i} F(q)^{2}\right)^{1 / 2} .
$$

Hereafter $X_{i} F$ denotes the directional derivative of the function $F$ along the vector field $X_{i}$.

\subsection{Sub-Riemannian minimal surfaces}

Let us compute the first variation of the horizontal area $A_{\Delta}(\cdot)$. Let $W \subset M$ be a smooth hypersurface. Take a bounded domain $\Omega \subset W$ and a vector field $V \in \operatorname{Vec}(M)$ such that $\left.V\right|_{\partial \Omega}=0$. For the moment we assume that $\Omega$ contains no characteristic points. Consider a one-parametric family of hypersurfaces generated by the vector field $V$

$$
\Omega^{t}=\mathrm{e}^{t V} \Omega, \quad \Omega^{0}=\Omega,
$$

and denote by $\nu^{t}$ the horizontal unit normals to $\Omega^{t}$. We have

$$
A_{\Delta}\left(\Omega^{t}\right)=\int_{\mathrm{e}^{t V} \Omega} i_{\nu^{t}} \mu=\int_{\Omega}\left(\mathrm{e}^{t V}\right)^{*} i_{\nu^{t}} \mu=\int_{\Omega} \mathrm{e}^{t L_{V}} i_{\nu^{t}} \mu,
$$

where $\mathrm{e}^{t L_{V}}: \Lambda^{k} M \rightarrow \Lambda^{k} M, k=0,1, \ldots$, is the operator on the space of forms defined as the unique solution of the operator Cauchy problem (see [2]):

$$
\frac{\mathrm{d}}{\mathrm{d} t}\left(P^{t}\right)=P^{t} \circ L_{V}, \quad P^{0}=\mathrm{Id} .
$$

Further,

$$
\left.\frac{\partial}{\partial t}\right|_{t=0} A_{\Delta}\left(\Omega^{t}\right)=\int_{\Omega} L_{V} i_{\nu} \mu+\int_{\Omega} i_{\left.\frac{\partial \nu t}{\partial t}\right|_{t=0} \mu .} \mu .
$$

The second integral in (1.7) vanishes because the horizontal vector field $\left.\frac{\partial \nu^{t}}{\partial t}\right|_{t=0}$ is tangent to $\Omega$. Indeed, at any non-characteristic point $q \in \Omega$ we have $\nu(q) \notin T_{q} \Omega$ and $\operatorname{dim} \Delta_{q} \cap T_{q} \Omega=n-2$. On the other hand it is not difficult to show that $\nu^{t}$ is smooth w.r.t. $t$ for $t$ sufficiently small (for instance, in can be derived from (1.6)). Thus differentiating the equality $\left\langle\nu^{t}, \nu^{t}\right\rangle_{\Delta}=1$ we get

$$
\left\langle\left.\frac{\partial \nu^{t}}{\partial t}\right|_{t=0}, \nu\right\rangle_{\Delta}=0
$$

and hence $\left.\frac{\partial \nu^{t}}{\partial t}\right|_{t=0}(q) \in T_{q} \Omega$. 
Using Cartan's formula we can transform the first part of (1.7) as follows:

$$
\int_{\Omega} L_{V} i_{\nu} \mu=\int_{\Omega}\left(i_{V} \circ d+d \circ i_{V}\right) i_{\nu} \mu=\int_{\Omega}\left(i_{V} \circ d \circ i_{\nu}\right) \mu+\int_{\Omega}\left(d \circ i_{V} \circ i_{\nu}\right) \mu .
$$

Applying the Stokes theorem to the second integral we see that it vanishes:

$$
\int_{\Omega}\left(d \circ i_{V} \circ i_{\nu}\right) \mu=\int_{\partial \Omega}\left(i_{V} \circ i_{\nu}\right) \mu=0
$$

provided $\left.V\right|_{\partial \Omega}=0$ and $\partial \Omega$ is sufficiently regular. Thus,

$$
\left.\frac{\partial}{\partial t}\right|_{t=0} A_{\Delta}\left(\Omega^{t}\right)=\int_{\Omega} i_{V}\left(d \circ i_{\nu} \mu\right)
$$

Definition 1.5. The hypersurface $W$ is called minimal w.r.t. the sub-Riemannian structure $\Delta$ (or just $\Delta$-minimal) if

$$
\left.\left(d \circ i_{\nu} \mu\right)\right|_{W \backslash \Sigma}=0
$$

where $\Sigma$ is the singular set of $W$.

Needless to say that property (1.9) does not depend on the chosen orientation in $M$. The whole construction can be further generalized for the case of vector distributions of co-rank greater than 1.

\subsection{Canonical form of the minimal surface equation in contact sub-Riemannian manifolds}

Up to now we were dealing with the general case of a sub-Riemannian manifold endowed with a co-rank 1 distribution. From now on we restrict ourselves to the case $n=2 m+1$ and assume that the distribution $\Delta$ is contact, i.e., the $2 m+1$-form $(d \omega)^{m} \wedge \omega$ is non-degenerate. In this case we say that $M$ is a contact subRiemannian manifold.

First of all we recall that in the contact case there exists a special uniquely defined vector field $X \in \operatorname{Vec}(M)$ associated with $\omega$. This vector field is called the Reeb vector field of the contact form $\omega$ and it satisfies the following equalities

$$
\omega_{q}(X(q))=1, \quad d_{q} \omega(v, X(q))=0, \quad \forall v \in \Delta_{q} .
$$

Using this vector field we can canonically extend the sub-Riemannian structure on $\Delta$ to the whole $T M$. The resulting Riemannian structure in $M$ is compatible with $\Delta$ by construction.

In what follows we set $X_{2 m+1} \equiv X$ and denote by $c_{i j}^{k} \in C^{\infty}(M)$ the structural constants of the frame $\left\{X_{i}\right\}_{i=1}^{2 m+1}$ :

$$
\left[X_{i}, X_{j}\right]=-\sum_{k=1}^{2 m+1} c_{i j}^{k} X_{k}
$$

Let $\left\{\theta_{i}\right\}_{i=1}^{2 m+1}$ be the basis of 1 -forms dual to $\left\{X_{i}\right\}_{i=1}^{2 m+1}$. Clearly, $\theta_{2 m+1} \equiv \omega$ and the canonical volume form reads

$$
\mu=\theta_{1} \wedge \ldots \wedge \theta_{2 m+1} .
$$

We also recall that from Cartan's formula it follows that

$$
d \theta_{k}=\sum_{\substack{i, j=1 \\ i<j}}^{2 m+1} c_{i j}^{k} \theta_{i} \wedge \theta_{j}, \quad k=1, \ldots, 2 m+1 .
$$


Now we are ready to derive the canonical form of the minimal surface equation (1.9) in the contact case. We have

$$
i_{\nu} \mu=\left(\sum_{k=1}^{2 m}(-1)^{k+1} \nu_{k} \theta_{1} \wedge \ldots \wedge \widehat{\theta_{k}} \wedge \ldots \wedge \theta_{2 m}\right) \wedge \theta_{2 m+1}=\Xi \wedge \theta_{2 m+1} .
$$

Here $\widehat{\theta_{k}}$ denotes the omitted element in the wedge product and

$$
\Xi=\sum_{k=1}^{2 m}(-1)^{k+1} \nu_{k} \theta_{1} \wedge \ldots \wedge \widehat{\theta_{k}} \wedge \ldots \wedge \theta_{2 m}
$$

Further,

$$
d i_{\nu} \mu=d \Xi \wedge \theta_{2 m+1}-\Xi \wedge d \theta_{2 m+1} .
$$

Recalling now that $d \nu_{k}=\sum_{i=1}^{2 m+1} X_{i} \nu_{k} \theta_{i}$, we get

$$
\begin{aligned}
d \Xi \wedge \theta_{2 m+1} & =\sum_{k=1}^{2 m}(-1)^{k+1}\left(d \nu_{k} \wedge \theta_{1} \wedge \ldots \wedge \widehat{\theta_{k}} \wedge \ldots \wedge \theta_{2 m}+\nu_{k} d\left(\theta_{1} \wedge \ldots \wedge \widehat{\theta_{k}} \wedge \ldots \wedge \theta_{2 m}\right)\right) \wedge \theta_{2 m+1} \\
& =\left(\sum_{k=1}^{2 m} X_{k} \nu_{k}+\sum_{j=1}^{2 m} \nu_{k} c_{k j}^{j}\right) \mu .
\end{aligned}
$$

On the other hand,

$$
\Xi \wedge d \theta_{2 m+1}=\Xi \wedge \sum_{\substack{i, j=1 \\ i<j}}^{2 m+1} c_{i j}^{2 m+1} \theta_{i} \wedge \theta_{j}=-\left(\sum_{k=1}^{2 m} \nu_{k} c_{k 2 m+1}^{2 m+1}\right) \mu .
$$

Summing up we obtain the following equation:

$$
\left.\left[\operatorname{div}^{\Delta} \nu+\sum_{i=1}^{2 m} \nu_{i}\left(\sum_{j=1}^{2 m+1} c_{i j}^{j}\right)\right]\right|_{W \backslash \Sigma}=0
$$

The left-hand side of (1.13) corresponds to the sub-Riemannian mean curvature of the hypersurface $W$, while its first term

$$
\operatorname{div}^{\Delta} \nu=\sum_{i=1}^{2 m} X_{i} \nu_{i}
$$

is called the horizontal divergence of the sub-Riemannian normal $\nu$. Equation (1.13) is the canonical subRiemannian minimal surface equation in a contact sub-Riemannian manifold.

Remark 1.6. In [7] there was introduced the notion of minimal surfaces associated with CR structures in pseudohermitian manifolds. This approach later was used in [6,8], and in [13]. In general the sub-Riemannian structures we consider in the present paper are not equivalent to the CR structures, and the class of surfaces satisfying (1.13) differs from its analog defined in [7]. Nevertheless, in many particular cases, for instance, in the cases of sub-Riemannian structures associated with the Heisenberg group, the group of roto-translations, and $S^{3}$, these structures coincide. Thus in these cases our results are comparable with the ones of cited papers. 
Example 1.7 (the Heisenberg distribution). Let $M=\mathbb{R}^{2 m+1}$ and denote by $\left(x_{1}, \ldots, x_{2 m}, t\right)=q$ the Cartesian coordinates in $M$. Let $\Delta$ be such that $\Delta_{q}=\operatorname{span}\left\{X_{i}(q)\right\}_{i=1}^{2 m}, q \in M$, where

$$
\begin{gathered}
X_{i}(q)=\partial_{x_{i}}-\frac{x_{i+m}}{2} \partial_{t}, \\
X_{i+m}(q)=\partial_{x_{i+m}}+\frac{x_{i}}{2} \partial_{t}, \quad i=1, \ldots, m .
\end{gathered}
$$

The vector distribution $\Delta$ is characterized by the following commutative relations:

$$
\left[X_{i}, X_{j}\right]=0 \text { for }|i-j| \neq m \text {, and }\left[X_{i}, X_{i+m}\right]=\partial_{t},
$$

and therefore it is a co-rank 1 bracket-generating distribution. The vector fields $X_{i}, i=1, \ldots, 2 m$, generate the Heisenberg Lie algebra in $\mathbb{R}^{2 m+1}$. In what follows we will call vector distributions, which satisfy the commutative relations (1.15), the Heisenberg distributions. One can show that the space $\mathbb{R}^{2 m+1}$ endowed with the structure of this distribution is the Heisenberg group $\mathbb{H}^{m}[14]$.

From (1.2) we find the canonical 1-form $\omega$ :

$$
\omega= \pm \frac{1}{\sqrt{m}}\left(d t-\frac{1}{2} \sum_{i=1}^{m}\left(x_{i} d x_{i+m}-x_{i+m} d x_{i}\right)\right) .
$$

Clearly $\omega$ is a contact form since $(d \omega)^{m} \wedge d \omega= \pm \frac{1}{m^{m}} \bigwedge_{i=1}^{2 m} d x_{i} \wedge d t$ is non-degenerate. The associated Reeb vector field is $X= \pm \sqrt{m} \partial_{t}$. The only non-zero structural constants of the canonical frame are $c_{i i+m}^{2 m+1}= \pm \frac{1}{\sqrt{m}}$, $i=1, \ldots, m$. Due to the high degeneracy of the sub-Riemannian structure the canonical minimal surface equation takes a very simple form:

$$
\left.\operatorname{div}^{\Delta} \nu\right|_{W \backslash \Sigma}=0
$$

This is the well known minimal surface equation in the Heisenberg group (see [7,10,12,16], etc.)

\section{Sub-Riemannian minimal surfaces associated with $(2,3)$ Contact VECTOR DISTRIBUTIONS}

The less dimensional situation where sub-Riemannian minimal surfaces appear is the case of a contact distribution $\Delta$ of rank 2 in the 3-dimensional manifold $M$. Almost all known results on sub-Riemannian minimal surfaces are related to this case and concern minimal surfaces in Lie groups mentioned above. In this paper we try to give a general picture. Our main idea is to study (1.13) using the classical method of characteristics. This point of view permits to describe all minimal surfaces, and, as we will show in a while, to solve the problem of the presence of the characteristic points. All this is possible due to the fact that $\operatorname{dim} T_{q} M \cap \Delta_{q}=1$ at any non-characteristic point $q \in M$.

\subsection{The $(2,3)$ structures}

From now on $n=3$ and $\Delta=\bigcup_{q \in M} \Delta_{q}$ with $\Delta_{q}=\operatorname{span}\left\{X_{1}(q), X_{2}(q)\right\}, q \in M$. As before, we complete the horizontal frame by the Reeb vector field $X \equiv X_{3}$ associated with $\Delta$, and denote by $c_{i j}^{k}$ the structural constant of the frame $\left\{X_{i}\right\}_{i=1}^{3}$. By definition, $c_{i j}^{k}=-c_{j i}^{k}$. Moreover, (1.10) and (1.11) imply

$$
c_{12}^{3}= \pm 1, \quad c_{13}^{3}=c_{23}^{3}=0 .
$$


Here the sign of $c_{12}^{3}$ is to be chosen in agreement upon the sign of $\omega$. More symmetry relations of the structural constants can be obtained from the Jacobi identity

$$
\left[X_{1},\left[X_{2}, X_{3}\right]\right]+\left[X_{3},\left[X_{1}, X_{2}\right]\right]+\left[X_{2},\left[X_{3}, X_{1}\right]\right]=0 .
$$

In particular, if $M$ is a Lie group, and $\left\{X_{i}\right\}_{i=1}^{3}$ is the associated basis of invariant vector fields, then the structural constants do not depend on the points of the base manifold $M$, and the Jacobi identity implies the following additional symmetry relations:

$$
c_{13}^{1}+c_{23}^{2}=0, \quad c_{12}^{1} c_{13}^{1}+c_{12}^{2} c_{23}^{1}=0, \quad c_{12}^{1} c_{13}^{2}+c_{12}^{2} c_{23}^{2}=0 .
$$

Let us now consider a regular hypersurface $W \subset M$ and let $\nu \in \Delta$ be its horizontal normal. As we know, $\nu$ is well defined on $W \backslash \Sigma$, where $\Sigma$ is the singular set of $W$, which contains characteristic points of $W$. If $W$ is $\Delta$-minimal, then by $(2.1)$,

$$
\left.\left(X_{1} \nu_{1}+X_{2} \nu_{2}+\nu_{1} c_{12}^{2}-\nu_{2} c_{12}^{1}\right)\right|_{W \backslash \Sigma}=0 .
$$

Assume that $W$ is given as a zero level set of a smooth function $F$. Then $\Sigma=\left\{q \in W \mid X_{1} F(q)=X_{2} F(q)=0\right\}$, and away from $\Sigma$ the function $F$ satisfies the following PDE:

$$
\begin{gathered}
{\left[\left(X_{1}^{2} F\left(X_{2} F\right)^{2}+X_{2}^{2} F\left(X_{1} F\right)^{2}-X_{1} F X_{2} F\left(X_{1} \circ X_{2}+X_{2} \circ X_{1}\right) F\right) D_{1}^{-3}+\right.} \\
\left.\left(c_{12}^{2} X_{1} F-c_{12}^{1} X_{2} F\right) D_{1}^{-1}\right]\left.\right|_{W \backslash \Sigma}=0, \quad D_{1}=\sqrt{\left(X_{1} F\right)^{2}+\left(X_{2} F\right)^{2}} .
\end{gathered}
$$

Some non-trivial solutions of this equation are known, especially in the particular case of $\mathbb{H}^{1}$, the interested reader can consult [7] and other papers from the bibliography. Let us also consider another important for applications case of the distribution associated with the Lie group $E_{2}$ (the group of rotations and translations of the plane).

Example 2.1. The Lie group $E_{2}$ can be realized as $\mathbb{R}^{2} \times \mathbb{S}^{1}$. In coordinates $q=(x, y, z)$, where $(x, y) \in \mathbb{R}^{2}$ and $z \in \mathbb{S}^{1}$, the left-invariant basis of the corresponding Lie algebra is given by vector fields

$$
X_{1}=\cos z \partial_{x}+\sin z \partial_{y}, \quad X_{2}=\partial_{z} .
$$

It is easy to check that the horizontal distribution $\Delta^{E_{2}}$ with sections $\Delta_{q}^{E_{2}}=\operatorname{span}\left\{X_{1}(q), X_{2}(q)\right\}, q \in M$, is contact, the corresponding canonical 1-form is $\omega= \pm(\sin z d x-\cos z d y)$. The Reeb vector field coincides with the Lie bracket $\left[X_{1}, X_{2}\right]$ (up to the sign) and the only non-zero structural constants are $c_{12}^{3}=c_{23}^{1}= \pm 1$. Therefore, the minimal surface equation, as in the Heisenberg case, contains only the divergence term:

$$
\left.\left(X_{1} \nu_{1}+X_{2} \nu_{2}\right)\right|_{W \backslash \Sigma}=0 .
$$

For instance one can easily check that the following surfaces are $\Delta^{E_{2}}$-minimal:
(a) $y=x+B(\sin z+\cos z)+C, \quad B, C=$ const.;
(b) $A x+B \sin z=C, \quad A, B, C=$ const.;
(c) $x \cos z+y \sin z=0$. 


\subsection{General structure of sub-Riemannian minimal surfaces and the method of characteristics}

Equation (2.4) is essentially degenerate and it is quite difficult to treat it by direct methods. Instead here we propose an alternative way to study its solutions using the classical method of characteristics. The first step in this direction is to pass from the sub-Riemannian normal $\nu$ to its $\Delta$-orthogonal complement. Namely, we denote by $\eta$ the horizontal unite vector field $\eta=\eta_{1} X_{1}+\eta_{2} X_{2} \in \Delta$ such that $\eta_{1}=\nu_{2}$ and $\eta_{2}=-\nu_{1}$. Clearly $\eta$ is well defined on $W \backslash \Sigma$. One can easily check that $\langle\nu, \eta\rangle_{\Delta}=0$ and $\eta(q) \in T_{q} W$ for all $q \in W \backslash \Sigma$. The vector field $\eta$ is called the characteristic vector field of $W$, its integral curves on $W$ are the characteristic curves of $W$. By definition, these curves are horizontal curves $t \rightarrow \gamma(t) \in W$ satisfying

$$
\dot{\gamma}(t) \in T_{\gamma(t)} W \cap \Delta_{\gamma(t)} \quad \forall t
$$

Since $\|\nu\|_{\Delta}=\|\eta\|_{\Delta}=1$, we can introduce a new scalar parameter $\varphi$ such that

$$
\cos \varphi=\eta_{1}, \quad \sin \varphi=\eta_{2}
$$

so that

$$
\eta=\cos \varphi X_{1}+\sin \varphi X_{2}
$$

Our further analysis is based on the following observation. Assume for the moment that the sub-Riemannian minimal surface $W$ contains no characteristic points. Then $\nu$ and $\eta$ are well defined, and (2.3) becomes

$$
-\sin \varphi X_{2} \varphi-\cos \varphi X_{1} \varphi=\cos \varphi c_{12}^{1}+\sin \varphi c_{12}^{2}
$$

The equation above is a quasilinear PDE and we can apply the classical method of characteristics to find its solutions. Indeed, denote by $q_{i}, i=1,2,3$ some local coordinates on $M$ and let $t \rightarrow\left(q_{1}(t), q_{2}(t), q_{3}(t)\right)$ be a smooth (at least $C^{1}$ ) curve. Along this curve $\dot{\varphi}=\sum_{i=1}^{3} \frac{\partial \varphi}{\partial q_{i}} \dot{q}_{i}$ with $\cdot=\frac{\mathrm{d}}{\mathrm{d} t}$. Substituting this expression into (2.6) we get the following system of ODE:

$$
\left\{\begin{aligned}
\dot{q} & =\eta(q) \\
\dot{\varphi} & =-\cos \varphi c_{12}^{1}(q)-\sin \varphi c_{12}^{2}(q) .
\end{aligned}\right.
$$

Clearly this system is equivalent to (2.6).

The described construction motivates the following geometric interpretation of the problem. Denote $\bar{M}=$ $\{\bar{q}=(q, \varphi) \mid q \in M, \varphi \in \mathbb{R}\}$. The space $\bar{M}$ is a trivialization of the horizontal spherical bundle $S_{\Delta} M$ over $M$ :

$$
S_{\Delta} M=\left\{(q, v) \mid q \in M, v \in \Delta_{q},\|v\|_{\Delta}=1\right\}
$$

By $\pi$ we denote the canonical projection $\bar{M} \rightarrow M$ and set $X_{4} \equiv \partial_{\varphi}$. Clearly, the Riemannian structure on $\bar{M}$ associated with the orthonormal frame $\left\{X_{i}\right\}_{i=1}^{4}$ is compatible with the sub-Riemannian structure on $\Delta$. Since $\left[X_{i}, X_{4}\right] \equiv 0$ for $i=1,2,3$, the non-zero structural constants of the extended frame are the same as the ones of the frame $\left\{X_{i}\right\}_{i=1}^{3}$.

Now consider the generalized characteristic vector field $V \in \operatorname{Vec}(\bar{M})$ :

$$
V=\cos \varphi X_{1}+\sin \varphi X_{2}+g X_{4}, \quad g=-c_{12}^{1} \cos \varphi-c_{12}^{2} \sin \varphi
$$

It is easy to see that $V$ is well defined everywhere on $\bar{M}$, it has no singular points, and it is not difficult to verify that it is invariant w.r.t. the choice of the horizontal basis on $\Delta$. Moreover, since $\pi_{*}[V(\bar{q})]=\eta(q)$ the projection of the integral curves of $V$ on $M$ are exactly the characteristics of equation (2.6). 
Let us take now a smooth vector field $\xi \in \operatorname{Vec}(\bar{M})$ and denote by $\Gamma$ its integral curve starting at $\bar{q}_{0}: \Gamma(s)=$ $\mathrm{e}^{s \xi} \bar{q}_{0}$. Further, consider

$$
\mathcal{W}=\left\{\bar{q}(t, s) \in \bar{M} \mid \bar{q}(t, s)=\left(\mathrm{e}^{t V} \circ \mathrm{e}^{s \xi}\right) \bar{q}_{0}, \quad t \in\left[-\varepsilon_{1}, \varepsilon_{2}\right], \quad s \in\left[-\delta_{1}, \delta_{2}\right]\right\}
$$

where $\delta_{i}$ and $\varepsilon_{i}, i=1,2$, are some positive numbers. By construction, $\mathcal{W}$ is the solution of the Cauchy problem

$$
\left\{\begin{array}{l}
\dot{\bar{q}}(t, s)=V(\bar{q}(t, s)) \\
\bar{q}(0, s)=\Gamma(s), \quad s \in\left[-\delta_{1}, \delta_{2}\right]
\end{array}\right.
$$

for $t \in\left[-\varepsilon_{1}, \varepsilon_{2}\right]$. The theorem of existence and uniqueness of solutions of ODE guarantees that this solution is unique since the manifold $M$ and the curve of initial conditions $\Gamma$ are smooth. Moreover, if $\left.\xi \wedge V\right|_{\Gamma} \neq 0$, then $\mathcal{W}$ locally has a structure of a 2-dimensional sub-manifold of $\bar{M}$. In our further considerations we will use the following:

Proposition 2.2. Let $\bar{q} \in \bar{M}$ and $\zeta=\sum_{i=1}^{4} \zeta_{i} X_{i}(\bar{q})$. Then $\zeta \wedge V(\bar{q})=0$ if and only if

$$
\begin{gathered}
\zeta_{3}=0, \\
\zeta_{1} \sin \varphi-\zeta_{2} \cos \varphi=0, \\
g(\bar{q}) \zeta_{1}=\zeta_{4} \cos \varphi, \quad g(\bar{q}) \zeta_{2}=\zeta_{4} \sin \varphi .
\end{gathered}
$$

Proof. The condition $\zeta \wedge V(\bar{q})=0$ means that

$$
\operatorname{dim} \operatorname{span}\{V(\bar{q}), \zeta\}<2 .
$$

In other words, all second order minors of the matrix

$$
\left(\begin{array}{cccc}
\zeta_{1} & \zeta_{2} & \zeta_{3} & \zeta_{4} \\
\cos \varphi & \sin \varphi & 0 & g(\bar{q})
\end{array}\right)
$$

are zero. Taking into account that the pair of conditions $\zeta_{3} \sin \varphi=0, \zeta_{3} \cos \varphi=0$ imply $\zeta_{3}=0$ we get (2.10), (2.11) and (2.12).

Conditions (2.10)-(2.12) admit a very clear geometrical interpretation. Indeed, needless to say that by construction any singular point of $\mathcal{W}$ of form (2.8) is a singular point of its projection $W=\pi[\mathcal{W}]$. On the other hand, at a regular point $\bar{q} \in \mathcal{W}$ conditions (2.10)-(2.12) cannot be satisfied simultaneously for all $\zeta \in T_{\bar{q}} \mathcal{W}$. In particular, if for some $\zeta \in T_{\bar{q}} \mathcal{W}(2.10)$ fails, then $\bar{q}$ is a regular point of $\mathcal{W}$, its projection $q=\pi[\bar{q}]$ is a regular point of the projected surfaces $W$, and $W$ satisfies equation (2.3) at $q$. On the other hand, it is easy to see that $q$ is a characteristic point of $W$ if and only if $\zeta_{3}=0$ for all $\zeta \in T_{\bar{q}} \mathcal{W}$. In addition, if (2.11) fails at $\bar{q}$ for some $\zeta$, then $\pi_{*}\left[T_{\bar{q}} \mathcal{W}\right]=\Delta_{q}$. If both conditions (2.10) and (2.11) are satisfied at $q$, then $T_{\bar{q}} \mathcal{W}=\operatorname{span}\left\{V(\bar{q}), \partial_{\varphi}\right\}$. In this case $\operatorname{dim} \operatorname{rank}\left\{\eta(q), \pi_{*}[\zeta]\right\}=1$ for all $\zeta \in T_{\bar{q}} \mathcal{W}$, and hence the characteristic point $q$ is a singular point of $W$.

Definition 2.3. Let $\mathcal{W} \subset \bar{M}, \operatorname{dim} \mathcal{W}=2$, be a smooth surface and let $W=\pi[\mathcal{W}]$. Assume $\bar{q} \in \mathcal{W}$ is such that $q=\pi[\bar{q}] \in \Sigma$. Then $q$ is

(a) a regular characteristic point of $W$ if

$$
\pi_{*}\left[T_{\bar{q}} \mathcal{W}\right]=\Delta_{q}
$$


(b) a singular characteristic point of $W$ if

$$
T_{\bar{q}} \mathcal{W}=\operatorname{span}\left\{V(\bar{q}), \partial_{\varphi}\right\}
$$

Summing up we see that the projected surface $W=\pi[\mathcal{W}]$ for the solutions of (2.9) is a $\Delta$-minimal surface, possibly with singularities of the described above types. Moreover, given a $\Delta$-minimal surface $W$ and a noncharacteristic point $q$ on it there always locally exists a 2-dimensional surface in $\bar{M}$ of form (2.8) projecting on it. Indeed, one can easily reconstruct $V$ and $\varphi$ using (1.6) and (2.5). In addition, since $q$ is non-singular, there exists a smooth curve $s \rightarrow \gamma(s) \in W$ containing $q$ and such that $\frac{\mathrm{d} \gamma(s)}{\mathrm{d} s} \wedge \eta(\gamma(s)) \neq 0$. So, solving (2.9) for $\Gamma(s)=(\gamma(s), \varphi(\gamma(s)))$, one finds a 2-dimensional surface (2.8) for some $\varepsilon_{i}, \delta_{i}, i=1,2$, which projects on $W$. Thus in the case of $(2,3)$ contact sub-Riemannian structures Definition 1.5 can be naturally generalized as follows:

Definition 2.4. Let $M, \operatorname{dim} M=3$, be a smooth contact sub-Riemannian manifold. We say that the hypersurface $W \in M$ is $\Delta$-minimal w.r.t. the sub-Riemannian structure $\Delta$ of co-rank 1 if it can be presented as the projection of the one-parametric family of solutions of the Cauchy problem (2.9) for some curve $\Gamma \in S_{\Delta} M$.

Notice, that in the sense of the above definition the characteristic vector field $\eta$ of a sub-Riemannian minimal surface $W$, being the projection of the generalized characteristic vector field $V$, is defined also on the singular set $\Sigma$, though it not true for the sub-Riemannian normal $\nu$.

In what follows we will call $\mathcal{W}$ and $\Gamma$ the generating surface and generating curve of the minimal surface $W=\pi[\mathcal{W}]$.

Remark 2.5. In a particular but important for the applications case of Lie groups the described method provides an explicit parameterization of the minimal surfaces. Indeed, recall that in the Lie group case the functions $c_{12}^{1}$ and $c_{12}^{2}$, associated with the basis of the invariant vector fields, are constants. So, for any fixed $s$ one can perform the direct integration of the second equation of (2.7). The obtained function $\varphi(t, s)$ can be used then to solve the first equation of (2.7). If, moreover,

$$
c_{12}^{1}=c_{12}^{2}=0,
$$

then $\varphi$ is constant along any characteristic curve. The resulting minimal surface is a kind of ruled surface, whose rulings are the characteristic curves.

Example 2.6. Consider the case of the Heisenberg group $\mathbb{H}^{1}$. Let us use the standard Cartesian coordinates $(x, y, z)$ in $\mathbb{R}^{3}$ so that the horizontal basis is given by

$$
X_{1}=\partial_{x}-\frac{y}{2} \partial_{z}, \quad X_{2}=\partial_{y}+\frac{x}{2} \partial_{z} .
$$

We fix the orientation by choosing the Reeb vector field $X_{3}=\partial_{z}$, so that the only non-zero structural constant is $c_{12}^{3}=-1$. Condition (2.13) is satisfied, and hence the parameter $\varphi$ is constant along characteristic curves. The characteristic vector field reads

$$
V=\cos \varphi \partial_{x}+\sin \varphi \partial_{y}+\frac{1}{2}(x \sin \varphi-y \cos \varphi) \partial_{z} .
$$

Thus any characteristic curve satisfies the following system of ODE for some fixed $\varphi$ :

$$
\dot{x}=\cos \varphi, \quad \dot{y}=\sin \varphi, \quad \dot{z}=\frac{1}{2}(x \sin \varphi-y \cos \varphi) .
$$


We easily see that characteristic curves are straight lines. The minimal surface generated by the curve $\Gamma(s)=$ $\left(x_{0}(s), y_{0}(s), z_{0}(s), \varphi(s)\right)$ admits the following parameterization:

$$
\begin{gathered}
x(t, s)=t \cos \varphi(s)+x_{0}(s), \quad y(t, s)=t \sin \varphi(s)+y_{0}(s), \\
z(t, s)=\frac{t}{2}\left(x_{0}(s) \sin \varphi(s)-y_{0}(s) \cos \varphi(s)\right)+z_{0}(s) .
\end{gathered}
$$

Example 2.7. In the case of the group of roto-translations $E_{2}$ condition (2.13) is satisfied as well, and $\varphi$ is constant along characteristics. The characteristic vector field is given by $V=\cos \varphi \cos z \partial_{x}+\cos \varphi \sin z \partial_{y}+$ $\sin \varphi \partial_{z}$. The characteristic curves are then the integral curves of the system:

$$
\dot{x}=\cos \varphi \cos z, \quad \dot{y}=\cos \varphi \sin z, \quad \dot{z}=\sin \varphi .
$$

The solution generated by the curve $\Gamma(s)=\left(x_{0}(s), y_{0}(s), z_{0}(s), \varphi(s)\right)$ has the form

$$
\begin{aligned}
& x(t, s)=\cos \left(t \sin \varphi(s)+z_{0}(s)\right) \cot \varphi(s)+x_{0}(s), \\
& y(t, s)=-\sin \left(t \sin \varphi(s)+z_{0}(s)\right) \cot \varphi(s)+y_{0}(s), \\
& z(t, s)=t \sin \varphi(s)+z_{0}(s)
\end{aligned}
$$

for all $s$ such that $\varphi(s) \neq 0, \pi$, and

$$
x(t, s)= \pm t \cos z_{0}(s)+x_{0}(s), \quad y(t, s)= \pm t \sin z_{0}(s)+y_{0}(s), \quad z(t, s)=z_{0}(s)
$$

for $s$ where $\varphi(s)=0 \bmod \pi$. It is not difficult to verify that this surface is smooth w.r.t. $s$. Observe that the characteristic curves, except those that correspond to $\varphi(s)=0 \bmod \pi$, are not straight lines.

\subsection{Local structure of singular sets of sub-Riemannian minimal surfaces}

Given a vector field $\xi \in \operatorname{Vec}(\bar{M})$ let us denote by $\xi^{t}=\sum_{i=1}^{4} \xi_{i}^{t} X_{i}=\mathrm{e}_{*}^{t V} \xi$ its push-forward by the characteristic flow $\mathrm{e}^{t V}$. Consider the parameterized surface $\mathcal{W} \in \bar{M}$ of form (2.8). As

$$
\frac{\partial}{\partial s} \bar{q}(t, s)=\left(\mathrm{e}_{*}^{t V} \xi\right)(\bar{q}(t, s))=\xi^{t}(\bar{q}(t, s))
$$

it follows that $\xi^{t}(\bar{q}) \in T_{\bar{q}} \mathcal{W}$ for all $\bar{q} \in \mathcal{W}$ and $t \in\left[-\varepsilon_{1}, \varepsilon_{2}\right]$. So, $\xi^{t}$ and $V$ form a basis on $T \mathcal{W}$.

We also observe that for any curve $\beta(s)=\bar{q}(t(s), s) \in \mathcal{W}$

$$
\frac{\mathrm{d} \beta(s)}{\mathrm{d} s}=t^{\prime}(s) V(\beta(s))+\xi^{t(s)}(\beta(s)) .
$$

If $\pi[\beta]$ contains a singular characteristic point of $W$, then it is tangent to the characteristic vector field at this point.

Our further analysis is based on the following Taylor's expansion of the components of the vector field $\xi^{t}$ along the integral curves of $V$. 
Proposition 2.8. Let $\xi \in \operatorname{Vec}(\bar{M})$ and consider the curve $\bar{q}_{t}=\mathrm{e}^{t V} \bar{q}$ starting at some point $\bar{q}=(q, \varphi) \in \bar{M}$. Then the functions $\alpha_{i}(t)=\xi_{i}^{t}\left(\bar{q}_{t}\right), i=1,2,3,4$, have the form

$$
\begin{aligned}
\alpha_{1}(t)= & \alpha_{1}(0)-t\left(c_{12}^{1}(q)\left(\alpha_{1}(0) \sin \varphi-\alpha_{2}(0) \cos \varphi\right)\right. \\
& \left.-\alpha_{3}(0)\left(c_{13}^{1}(q) \cos \varphi+c_{23}^{1}(q) \sin \varphi\right)+\alpha_{4}(0) \sin \varphi\right)+o\left(t^{2}\right), \\
\alpha_{2}(t)= & \alpha_{2}(0)-t\left(c_{12}^{2}(q)\left(\alpha_{1}(0) \sin \varphi-\alpha_{2}(0) \cos \varphi\right)\right. \\
& \left.-\alpha_{3}(0)\left(c_{13}^{2}(q) \cos \varphi+c_{23}^{2}(q) \sin \varphi\right)-\alpha_{4}(0) \cos \varphi\right)+o\left(t^{2}\right), \\
\alpha_{3}(t)= & \alpha_{3}(0)+t\left(\alpha_{1}(0) \sin \varphi-\alpha_{2}(0) \cos \varphi\right) \\
& -\frac{t^{2}}{2}\left(\alpha_{4}(t)+c_{12}^{1}\left(q_{t}\right) \alpha_{1}(t)+c_{12}^{2}\left(q_{t}\right) \alpha_{2}(t)+o(t)\right) .
\end{aligned}
$$

Proof. The proof of the proposition consists in a straightforward computation. We give just a sketch of it for $\alpha_{3}(t)$, the remaining formulae can be derived in the same way.

Recall that the push-forward operator admits the following representation (see $[1,2])$ :

$$
\mathrm{e}_{*}^{t V} \xi=\mathrm{e}^{-\operatorname{tad} V} \xi=\left(I d-t[V, \cdot]+\frac{t^{2}}{2 !}[V,[V, \cdot]]+\ldots\right) \xi, \quad \xi \in \operatorname{Vec}(\bar{M}) .
$$

Let us compute explicitly the first terms of this expansion. We have

$$
\begin{gathered}
{[V, \xi]=\left(V \xi_{1}+c_{12}^{1}\left(\xi_{1} \sin \varphi-\xi_{2} \cos \varphi\right)-\xi_{3}\left(c_{13}^{1} \cos \varphi+c_{23}^{1} \sin \varphi\right)+\xi_{4} \sin \varphi\right) X_{1}} \\
+\left(V \xi_{2}+c_{12}^{2}\left(\xi_{1} \sin \varphi-\xi_{2} \cos \varphi\right)-\xi_{3}\left(c_{13}^{2} \cos \varphi+c_{23}^{2} \sin \varphi\right)-\xi_{4} \cos \varphi\right) X_{2} \\
+\left(V \xi_{3}-\left(\xi_{1} \sin \varphi-\xi_{2} \cos \varphi\right)\right) X_{3}+\left(V \xi_{4}-\xi g\right) X_{4} .
\end{gathered}
$$

This expression can be used in order to calculate the second order brackets. In particular, after all necessary simplifications for the third component we obtain

$$
[V,[V, \xi]]_{3}=V^{2} \xi_{3}-2 V\left(\xi_{1} \sin \varphi-\xi_{2} \cos \varphi\right)-\left(\xi_{4}+c_{12}^{1} \xi_{1}+c_{12}^{2} \xi_{2}\right)
$$

Observe that for any smooth function $f$

$$
f-t V f+\frac{t^{2}}{2 !} V^{2} f+\ldots=\mathrm{e}^{-t V} f
$$

where, by definition, for any $\bar{q} \in \bar{M}$ one has $\left(\mathrm{e}^{-t V} f\right)(\bar{q})=f\left(\mathrm{e}^{-t V} \bar{q}\right)$ (see [2]). Therefore

$$
\xi_{3}^{t}=\mathrm{e}^{-t V} \xi_{3}+t \mathrm{e}^{-t V}\left(\xi_{1} \sin \varphi-\xi_{2} \cos \varphi\right)-\frac{t^{2}}{2}\left(\xi_{4}+c_{12}^{1} \xi_{1}+c_{12}^{2} \xi_{2}\right)+o\left(t^{3}\right) .
$$

Taking into account that

$$
\left(\mathrm{e}^{-t V} f\right)\left(\bar{q}_{t}\right)=f\left(\mathrm{e}^{-t V} \bar{q}_{t}\right)=f(\bar{q}),
$$

and recalling that by definition $\alpha_{3}(t)=\xi_{3}^{t}\left(\mathrm{e}^{t V} \bar{q}\right)$ and $\xi_{i}(\bar{q})=\alpha_{i}(0)$, we get the desired expression:

$$
\alpha_{3}(t)=\alpha_{3}(0)+t\left(\alpha_{1}(0) \sin \varphi-\alpha_{2}(0) \cos \varphi\right)-\frac{t^{2}}{2}\left(\alpha_{4}(t)+c_{12}^{1}\left(q_{t}\right) \alpha_{1}(t)+c_{12}^{2}\left(q_{t}\right) \alpha_{2}(t)\right)+o\left(t^{3}\right) .
$$

The formulae for $\alpha_{1}(t)$ and $\alpha_{2}(t)$ can be derived in the same way. 
We are now ready to describe the structure of a sub-Riemannian minimal surface close to its characteristic point. Let the generating surface $\mathcal{W}$ be as in (2.8) for some $\xi \in \operatorname{Vec}(\bar{M})$ which satisfy the non-degeneracy condition

$$
\left.\xi \wedge V\right|_{\bar{q}(s)} \neq 0
$$

where $\bar{q}(s)=\mathrm{e}^{s \xi} \bar{q}_{0}, s \in\left[-\delta_{1}, \delta_{2}\right]$, is the integral curve of $\xi$ passing through $\bar{q}_{0}$. The point $q=\pi[\bar{q}(t, s)]$ is a characteristic point of $W=\pi[\mathcal{W}]$ if and only if

$$
\phi(t, s)=\xi_{3}^{t}\left(\mathrm{e}^{t V} \bar{q}(s)\right)=\phi_{0}(s)+t \phi_{1}(s)-\frac{t^{2}}{2} \phi_{2}(t, s)=0,
$$

where $\phi_{0}(s)=\xi_{3}(\bar{q}(s)), \phi_{1}(s)=\left(\xi_{1} \sin \varphi-\xi_{2} \cos \varphi\right)(\bar{q}(s))$, and $\phi_{2}(t, s)=\left(\xi_{4}+c_{12}^{1} \xi_{1}+c_{12}^{2} \xi_{2}+t f\right)(\bar{q}(t, s))$. In the last formula the function $f$ contains the higher order terms of the expansion (2.18).

If $\phi \neq 0$ on $I=\left[-\varepsilon_{1}, \varepsilon_{2}\right] \times\left[-\delta_{1}, \delta_{2}\right]$, then $W$ is a sub-Riemannian minimal surface without characteristic points. Assume now that $W$ contains a characteristic point. Without loss of generality we may assume that this point is $q_{0}=\pi[\bar{q}(0,0)]$, i.e., $\phi(0,0)=\phi_{0}(0)=0$.

I. Generic case. Recall that a generic property in the space $C^{r}(I ; \mathbb{R})$ is the property satisfied in an open dense subset of $C^{r}(I ; \mathbb{R})$. In particular, it is easy to see that generically a function $\phi \in C^{1}(I ; \mathbb{R})$, satisfying $\phi(0,0)=0$, has no singular point at $(0,0)$, i.e. ${ }^{2}$,

$$
d \phi(0,0) \neq 0 .
$$

In view of Definition 2.3, at $q_{0}$ we have to distinguish between the following two situations:

$$
\phi_{0}(0)=0,\left.\quad \frac{\partial \phi}{\partial t}\right|_{(0,0)}=\phi_{1}(0) \neq 0 ;
$$

and

$$
\phi_{0}(0)=0,\left.\quad \frac{\partial \phi}{\partial s}\right|_{(0,0)}=\phi_{0}^{\prime}(0) \neq 0,\left.\quad \frac{\partial \phi}{\partial t}\right|_{(0,0)}=\phi_{1}(0)=0 .
$$

Notice, that conditions (2.22) and (2.23) are conditions on the velocity of the generating curve $\Gamma$ at $\bar{q}_{0}$ and they are invariant w.r.t. regular reparameterizations of $\Gamma$. According to Definition 2.3, (2.22) describes a regular characteristic point, while (2.23) corresponds to a singular characteristic point.

Let us first consider case (2.22). We have

$$
\phi(0,0)=0, \quad \frac{\partial \phi}{\partial t}(0,0)=\phi_{1}(0) \neq 0 .
$$

By the implicit function theorem in a small neighborhood of the origin in $\mathbb{R}^{2}$ there exists a unique curve $t=t(s)$ such that $t(0)=0$ and $\phi(t(s), s)=0$. By construction, the curve $q(t(s), s) \in W$ consists of regular characteristic points, we will call such a curve a simple singular curve of $W^{3}$.

Now let us consider in detail the situation described by (2.23). Let us introduce two functions $\tilde{\phi}_{0}$ and $\tilde{\phi}_{1}$ such that $\phi_{0}(s)=s \tilde{\phi}_{0}(s)$ and $\phi_{1}(s)=s \tilde{\phi}_{1}(s)$, and, in addition, $\tilde{\phi}_{0}(0) \neq 0$. Observe that $\tilde{\phi}_{0}(0)=\left.\frac{\mathrm{d}}{\mathrm{d} s} \xi_{3}(\bar{q}(s))\right|_{s=0}$, i.e., (2.23) means that locally the projection of the generating curve contains no other characteristic points besides $q_{0}$.

\footnotetext{
${ }^{2}$ This simple fact can be also derived from the the Weak Transversality theorem: indeed, for $\phi \in C^{1}(I ; \mathbb{R})$ the condition $d \phi(0,0) \neq 0$ means that $\phi$ is transversal to $\{0\} \subset \mathbb{R}$, which implies that $\phi$ belongs to an open dense subset $C^{1}(I ; \mathbb{R})[3]$.

${ }^{3}$ In the language of the singularity theory the simple singular curves are resolvable singularities of sub-Riemannian minimal surfaces.
} 
Theorem 2.9. Let $\bar{q}_{0}$ be a regular point of a generating surface $\mathcal{W}$ of form (2.8) for $\xi \in$ Vec $\bar{M}$. Assume $q_{0}=\pi\left[\bar{q}_{0}\right]$ is a singular characteristic point of $W=\pi[\mathcal{W}]$, and (2.21) holds true. Then:

(a) a small enough neighborhood of $q_{0}$ in $W$ contains a pair of simple singular curves $\gamma_{ \pm}$so that $\gamma=$ $\gamma_{-} \cup\left\{q_{0}\right\} \cup \gamma_{+}$is a smooth (at least $C^{1}$ ) curve on $W$;

(b) there exists a choice of local coordinates $(\tau, \sigma) \in \Omega \subseteq \mathbb{R}^{2}$ such that

$$
W=\left\{q(\tau, \sigma)=\left(\tau^{2}, \sigma, \tau \sigma\right), \quad \tau, \sigma \in \Omega \subseteq \mathbb{R}^{2}\right\}, \quad q(0,0)=q_{0},
$$

i.e., in the neighborhood of $q_{0} W$ has the structure of Whitney's umbrella.

Proof. Denote $\zeta=\xi\left(\bar{q}_{0}\right)$. Observe that since $\bar{q}_{0}=\bar{q}(0,0)$ is a regular point $\phi_{2}(0,0) \neq 0$. Indeed, otherwise we would have $\zeta_{4}=-c_{12}^{1} \zeta_{1}-c_{12}^{2} \zeta_{2}$ and $\zeta_{1} \sin \varphi=\zeta_{2} \cos \varphi$. It is not difficult to see that these two conditions are equivalent to $(2.12)$, and this contradicts the regularity assumption on the initial point $\bar{q}_{0}$.

In order to prove part (a) let us treat the implicit equation $\phi(t, s)=0$ as a quadratic equation w.r.t. $t$-variable. The discriminant of this equation is given by the function

$$
D(t, s)=s\left(s \tilde{\phi}_{1}^{2}(s)+2 \tilde{\phi}_{0}(s) \phi_{2}(t, s)\right)=s \beta(t, s),
$$

where $D(0,0)=0$ and $\beta(0,0) \neq 0$. We have $\frac{\partial}{\partial t} D(0,0)=0$ and $\frac{\partial}{\partial s} D(0,0)=\beta(0,0) \neq 0$. Observe also that $D(t, 0) \equiv 0$. Thus we can assume $D(t, s)>0$ in a small neighborhood of $(0,0)$ for $s>0$. Indeed, this condition can be always satisfied by an appropriate choice of the sign of the parameter $s$. Now we obtain two implicit equations

$$
t=\frac{s \tilde{\phi}_{1}(s) \pm \sqrt{s \beta(t, s)}}{\phi_{2}(t, s)}
$$

which describe zero level sets of two functions

$$
\Phi^{ \pm}(t, s)=t-\frac{s \tilde{\phi}_{1}(s) \pm \sqrt{s \beta(t, s)}}{\phi_{2}(t, s)} .
$$

We have

$$
\Phi^{ \pm}(0,0)=0, \quad \frac{\partial}{\partial t} \Phi^{ \pm}(0,0)=1 .
$$

Applying the implicit function theorem to each of the functions $\Phi^{ \pm}$we see that in a small enough neighborhood of the origin in the half-plane $s \geq 0$ of the $(t, s)$-plane there exist two curves $\left(t^{ \pm}(s), s\right)$ satisfying (2.24). Since $t^{ \pm}(0)=0$ these curves meet each other at the origin of the $(t, s)$-plane. Since $\lim _{s \rightarrow 0} \frac{\partial}{\partial s} \Phi^{ \pm}(0, s)=\mp \infty$ they are both tangent to the $t$-axis. The corresponding curves on $W$ form a unique curve

$$
\gamma=\gamma_{-} \cup\left\{q_{0}\right\} \cup \gamma_{+}
$$

where $\gamma_{ \pm}(s)=q\left(t^{ \pm}(s), s\right), s>0$. The curve $\gamma$ is smooth at $q_{0}$, actually, in view of (2.17), it is tangent to the characteristic passing through $q_{0}$.

We claim that the short enough pieces of curves $\gamma_{ \pm}$contain only regular characteristic points. In order to prove this we show that the function $\chi=\xi_{1}^{t} \sin \varphi-\xi_{2}^{t} \cos \varphi$ is different from zero in a small neighborhood of $\bar{q}_{0}$. First of all we observe that for $s$ fixed

$$
\begin{aligned}
& \sin \varphi(t, s)=\sin \left(\varphi(s)+\operatorname{tg}(s)+o\left(t^{2} ; s\right)\right)=\sin \varphi(s)+\operatorname{tg}(s) \cos \varphi(s)+o\left(t^{2} ; s\right), \\
& \cos \varphi(t, s)=\cos \left(\varphi(s)+t q(s)+o\left(t^{2} ; s\right)\right)=\cos \varphi(s)-\operatorname{tg}(s) \sin \varphi(s)+o\left(t^{2} ; s\right) .
\end{aligned}
$$

Hereafter we denote $\varphi(s)=\varphi(0, s), \xi_{i}(s)=\xi_{i}(\bar{q}(0, s)), g(s)=g(\bar{q}(0, s))$, and $\chi(t, s)=\chi(\bar{q}(t, s))$. 
Without loss of generality we can assume that $\zeta_{1}=\zeta_{2}=0$. We have $\chi(0,0)=0$, and by definition, $\chi(0, s)=s \tilde{\phi}_{1}(s)$. Using (2.18) we obtain

$$
\left.\frac{\partial \chi}{\partial t}\right|_{(0,0)}=\frac{\partial}{\partial t}\left(\xi_{1}^{t} \sin \varphi-\xi_{2}^{t} \cos \varphi\right)\left(\bar{q}_{0}\right)=\left(\zeta_{4}-g(0)\left(\zeta_{1} \cos \varphi(0)+\zeta_{2} \sin \varphi(0)\right)\right) \neq 0,
$$

because, by assumption, $\bar{q}_{0}$ is a regular point of $\mathcal{W}$. Hence function $\chi$ can be zero only along the generating curve, i.e., the singular points should necessarily belong to the curve $q(s)$. But this is impossible since $q(s)$ does not contain other characteristic points besides $q_{0}$ due to (2.21). Therefore there is a small neighborhood of $q_{0}$ in $W$ which contains no other singular points besides $q_{0}$, and in particular the points $q\left(t^{ \pm}(s), s\right)$ for sufficiently small $s>0$ are regular characteristic points.

Let us prove part (b). The sub-Riemannian minimal surfaces are the images of the map $q: I \subseteq \mathbb{R}^{2} \rightarrow M$ such that $q(t, s)=\pi[\bar{q}(t, s)] \in M$. Again we assume that $\zeta_{i}=0, i=1,2$. We have

$$
\left.\frac{\partial q}{\partial t}\right|_{(0,0)}=\left(\cos \varphi X_{1}+\sin \varphi X_{2}\right)(q(0,0)),\left.\quad \frac{\partial q}{\partial s}\right|_{(0,0)}=\pi_{*}[\zeta]=0 .
$$

In order to prove (b), according to the well-known result by Whitney [17], it is enough to show that the vectors $v_{1}=\left.\frac{\partial q}{\partial t}\right|_{(0,0)}, v_{2}=\left.\frac{\partial^{2} q}{\partial t \partial s}\right|_{(0,0)}$ and $v_{3}=\left.\frac{\partial^{2} q}{\partial s^{2}}\right|_{(0,0)}$ are linearly independent. We have

$$
\begin{aligned}
\frac{\partial^{2} q(t, s)}{\partial t \partial s}= & \varphi_{s}^{\prime}(t, s)\left(-\sin \varphi(s) X_{1}(q(t, s))+\cos \varphi(t, s) X_{2}(q(t, s))\right) \\
& +\cos \varphi(t, s) \frac{\partial}{\partial s}\left(X_{1}(q(t, s))\right)+\sin \varphi(t, s) \frac{\partial}{\partial s}\left(X_{2}(q(t, s))\right) .
\end{aligned}
$$

By (2.26), the last two terms on the expression above vanish at $q_{0}=q(0,0)$. So,

$$
v_{2}=\zeta_{4}\left(-\sin \varphi X_{1}+\cos \varphi X_{2}\right)(q(0,0))
$$

since $\varphi_{s}^{\prime}(s)=\xi_{4}(s)$. Analogously we get

$$
v_{3}=\left(\frac{\mathrm{d} \xi_{1}}{\mathrm{~d} s} X_{1}+\frac{\mathrm{d} \xi_{2}}{\mathrm{~d} s} X_{2}+\frac{\mathrm{d} \xi_{3}}{\mathrm{~d} s} X_{3}\right)(q(0,0))
$$

An easy calculation shows that $\operatorname{det}\left\{v_{1}, v_{2}, v_{3}\right\}=\zeta_{4} \frac{\mathrm{d}}{\mathrm{d} s} \xi_{3}(0) \neq 0$.

II. Non-generic cases. It is worth to say a couple of words about what may happen in some degenerate situations, i.e., when $\phi$ is singular at $(0,0)$.

Consider first the case $\phi_{0}(0)=0, \phi_{1}(0)=\phi_{0}^{\prime}(0)=0$, and $\phi_{0}^{\prime \prime}(0) \neq 0$. Then we can set $\phi_{0}(s)=s^{2} \tilde{\phi}_{0}(s)$. Now, repeating the same steps as in the proof of Theorem 2.9, we get a pair of implicit functions:

$$
\widetilde{\Phi}^{ \pm}(t, s)=t-\frac{s \tilde{\phi}_{1}(s) \pm s \sqrt{\tilde{\beta}(t, s)}}{\phi_{2}(t, s)} .
$$


The implicit function theorem applied to both functions $\widetilde{\Phi}^{ \pm}$implies the existence of two pairs of curves $q\left(t^{ \pm}(s), s\right)$ in a small enough neighborhood of $s=0$. Notice that the concatenations

$$
\gamma^{ \pm}(s)=\left.\left.q\left(t^{ \pm}(s)\right)\right|_{s<0} \cup\left\{q_{0}\right\} \cup q\left(t^{ \pm}(s)\right)\right|_{s>0}
$$

are smooth and both tangent to the characteristic passing through $q_{0}$.

Concerning the degeneracies of higher order we observe that the local behavior of the singular curves in a neighborhood of a singular point is determined by the behavior of the function $t(s) \sim s^{k / 2}$ in the proximity of the singularity. So, for any finite $m_{1}>2$ and $m_{2}>1$, the structure of the singular curves in the case $\phi_{0}^{m_{1}} \neq 0$, $\phi_{1}^{m_{2}} \neq 0$ will be analogous to one of the two situations (2.25) and (2.27) described above. The rigorous proof in each singular case can be done in the same manner as above modulo a suitable modification, but this lies behind the scope of this paper.

To conclude this discussion we have to consider the following two particularly degenerate cases. Namely, if $\phi_{0}=\phi_{1} \equiv 0$, the generating curve $\Gamma_{*}$ is everywhere tangent to the plane $\operatorname{span}\left\{\partial_{\varphi}, V\right\}$, though (2.20) may be still verified. The projected curve $\gamma_{*}=\pi\left[\Gamma_{*}\right]$ consists of singular characteristic points (strongly singular curve), and any narrow enough stripe along $\gamma_{*}$ contains no other characteristic points of $W$. Indeed, the equation $\phi(t, s)=0$ has only trivial solution $t=0$ and moreover, $\phi(t, s) \neq 0$ for small enough $t>0$ since $\phi_{2}(0,0) \neq 0$.

The sub-Riemannian minimal surface generated by a purely "vertical curve" $\bar{q}(s)=\left(q_{0}, \varphi(s)\right)$ passing through $\bar{q}_{0}$ contains an isolated singular point $q_{0}$. In this case the whole strongly singular curve $\gamma_{*}$ collapses into a single point $q_{0}=\pi\left[\bar{q}_{0}\right]$. The same argument as before, after an obvious modification, implies that $q_{0}$ is an isolated singular point. Since, by assumption, $\bar{q}_{0}$ is a regular point of $\mathcal{W}$, the component $\xi_{4}(s)=\varphi^{\prime}(s) \neq 0$. The characteristic vector $\eta\left(q_{0}\right)$ "rotates" monotonically in the plane $T_{q(0,0)} W$. Moreover, if we consider a closed curve $\gamma(s)=\pi\left[\mathrm{e}^{\varepsilon V} \bar{q}(s)\right]$ for some fixed and sufficiently small $\varepsilon$, then to a complete revolution of the point along $\gamma$ there corresponds one complete revolution of the vector $\eta(\gamma(s))$. In particular, it follows that the index of the isolated singular point is +1 [4].

We now conclude this section by the following classification of characteristic points of sub-Riemannian minimal surfaces:

Let $\mathcal{W} \in \bar{M}$ be as in (2.8) and assume (2.20). Let $\bar{q}_{0}$ is be a regular point of $\mathcal{W}$ such that $q_{0}=\pi\left[\bar{q}_{0}\right]$ is a characteristic point of the projected surface $W=\pi[\mathcal{W}]$. Then in a small neighborhood of $q_{0} \in W$ there realizes one of the following situations:

- $q_{0}$ is a regular point of $W$. In a small enough neighborhood of $q$ the surface $W$ contains a unique simple singular curve passing through $q_{0}$;

- $q_{0}$ is a singular point of $W$. In addition,

- it can be an isolated singular characteristic point;

- in a small neighborhood of $q_{0}$ in $W$ there is a strongly singular curve passing through $q_{0}$;

- in a small neighborhood of $q_{0}$ in $W$ there is a smooth curve $\gamma$, which contains $q_{0}$ and has a common tangent with the characteristic passing through this point. This curve has form (2.25), and its branches $\gamma^{ \pm}$consist of regular characteristic points;

- in a small enough neighborhood of $q_{0}$ the surface $W$ contains a pair of curves of form (2.25), which are both tangent to the characteristic passing through $q_{0}$ and contain no other singular points besides $q_{0}$.

\section{EXAMPLE: SingUlar SETS OF SUB-RIEMANNIAN MiNimAL SURFACES IN $\mathbb{H}^{1}$}

We now illustrate the results of the previous section by examples of minimal surfaces associated with the Heisenberg distribution in $\mathbb{H}^{1}$. Some of the facts that will be discussed below were already noticed in $[6,7]$. We limit ourselves to consider only the sub-Riemannian minimal surfaces generated by smooth generating curves. Due to the explicit parameterization (2.15) knowing the generating curve $\Gamma(s), s \in \mathbb{R}$, is enough to reconstruct the whole projected surface together with its singular set. 
First of all observe that in the $\mathbb{H}^{1}$ case the Lie brackets of order greater than 2 of the generalized characteristic vector field $V$ with the vector fields $X_{1}, X_{2}$ and $X_{3}$ vanish. This implies that for any vector field $\xi \in \operatorname{Vec}(\bar{M})$ the right-hand sides of (2.18) contain at most quadratic terms w.r.t. $t$. More precisely,

$$
\begin{aligned}
& \alpha_{1}(t)=\xi_{1}(\bar{q})-t \xi_{4}(\bar{q}) \sin \varphi(\bar{q}), \quad \alpha_{2}(t)=\xi_{2}(\bar{q})+t \xi_{4}(\bar{q}) \cos \varphi(\bar{q}), \\
& \alpha_{3}(t)=\xi_{3}(\bar{q})+t\left(\xi_{1}(\bar{q}) \sin \varphi(\bar{q})-\xi_{2}(\bar{q}) \cos \varphi(\bar{q})\right)-\frac{t^{2}}{2} \xi_{4}(\bar{q}), \\
& \alpha_{4}(t)=\xi_{4}(\bar{q}) .
\end{aligned}
$$

Moreover, $\zeta \wedge V(\bar{q})=0$ for $\zeta \in T_{\bar{q}} M$ if and only if

$$
\zeta_{1} \sin \varphi-\zeta_{2} \cos \varphi=0, \quad \zeta_{3}=\zeta_{4}=0 .
$$

Proposition 3.1. Let $s \rightarrow \Gamma(s)$ be an integral curve of a smooth vector field $\xi \in \operatorname{Vec}(\bar{M})$ such that $\left.\xi \wedge V\right|_{\Gamma} \neq 0$. Let $W$ be a sub-Riemannian minimal surface generated by $\Gamma$. Then if for some $\hat{s}$

$$
2 \xi_{3} \xi_{4}+\left.\left(\xi_{1} \sin \varphi-\xi_{2} \cos \varphi\right)^{2}\right|_{\Gamma(\hat{s})}=0, \quad \text { and } \quad \xi_{4}(\hat{s}) \neq 0
$$

then the point $q(\hat{t}, \hat{s})$ is a singular point of $W$ for

$$
\hat{t}=\left.\frac{\xi_{1} \sin \varphi-\xi_{2} \cos \varphi}{\xi_{4}}\right|_{\Gamma(\hat{s})} .
$$

Moreover, the singular points of the type $(\hat{t}, \hat{s})$, where $\hat{t}$ is defined by $(3.3)$, are the only singular points of $W$.

Proof. The point $q_{*}=\pi[\bar{q}(\hat{t}, \hat{s})]$ is a singular point of $W$ iff

$$
\left.\operatorname{dim} \operatorname{span}\left\{\pi_{*}\left[\xi^{\hat{t}}\right], \eta\right\}\right|_{q_{*}}<2
$$

i.e.,

$$
\begin{gathered}
\cos \varphi(\hat{s}) \alpha_{2}(\hat{t} ; \hat{s})-\sin \varphi(\hat{s}) \alpha_{1}(\hat{t} ; \hat{s})=\left(\cos \varphi(\hat{s}) \xi_{2}(\hat{s})-\sin \varphi(\hat{s}) \xi_{1}(\hat{s})\right)+t \xi_{4}(\hat{s})=0, \\
\cos \varphi(\hat{s}) \alpha_{3}(\hat{t} ; \hat{s})=0, \quad \sin \varphi(\hat{s}) \alpha_{3}(\hat{t} ; \hat{s})=0 .
\end{gathered}
$$

Taking into account (3.1), one can easily see that (3.2) and (3.3) imply (3.4) and vice versa.

Remark 3.2. The role of the condition $\xi_{4}(\hat{s}) \neq 0$ in (3.2) becomes clear from the following observation. If $\xi_{4}(\hat{s})=0$ and $\cos \varphi(\hat{s}) \xi_{2}(\hat{s})-\sin \varphi(\hat{s}) \xi_{1}(\hat{s})=0$ for some $\hat{s}$, then $\xi_{3}(\hat{s}) \neq 0$ by the non-degeneracy assumption $\left.\xi \wedge V\right|_{\Gamma} \neq 0$, and hence $q(t, \hat{s})$ is a regular point of $W$ for any $t$.

The left-hand side of (3.2) is the discriminant

$$
D(s)=\left(\xi_{1}(s) \sin \varphi(s)-\xi_{2}(s) \cos \varphi(s)\right)^{2}+2 \xi_{3}(s) \xi_{4}(s)
$$

of the quadratic equation

$$
\xi_{3}(s)+t\left(\xi_{1}(s) \sin \varphi(s)-\xi_{2}(s) \cos \varphi(s)\right)-\frac{t^{2}}{2} \xi_{4}(s)=0
$$

which describes the characteristic points of $W$. If $\xi_{4}(s) \neq 0$, then it has at most 2 real roots:

$$
t_{*}^{ \pm}(s)=\frac{\xi_{1}(s) \sin \varphi(s)-\xi_{2}(s) \cos \varphi(s) \pm \sqrt{D(s)}}{\xi_{4}(s)}
$$


provided $D(s) \geq 0$. According to Proposition 3.1, the singular points are characterized by the roots of the equation $D(s)=0$. The two branches of simple singular curves described in Theorem 2.9 have the form

$$
\begin{gathered}
x_{*}^{ \pm}(s)=t_{*}^{ \pm}(s) \cos \varphi(s)+x_{0}(s), \quad y_{*}^{ \pm}(s)=t_{*}^{ \pm}(s) \sin \varphi(s)+y_{0}(s), \\
z_{*}^{ \pm}(s)=\frac{t_{*}^{ \pm}(s)}{2}\left(x_{0}(s) \sin \varphi(s)-y_{0}(s) \cos \varphi(s)\right)+z_{0}(s)
\end{gathered}
$$

and are defined for $s$ such that $D(s)>0$.

Example 3.3. The curve $\Gamma(s)=(0,0, s, s)$ for $s \in \mathbb{R}$ generates the counter-clockwise helicoid $q(t, s)=$ $(t \cos s, t \sin s, s)$, whose rulings are parallel to the $(x, y)$-plane. Since $\xi_{1}(s)=\xi_{2}(s)=0$ and $\xi_{3}(s)=\xi_{4}(s)=1$, we found that $D(s)=2$. Hence the singular set consists of two simple singular curves $( \pm \sqrt{2} \cos s, \pm \sqrt{2} \sin s, s)$ which never meet each other (Fig. 1). It is easy to see that all counter-clockwise helicoids have the same structure of singular set. Notice, that the clockwise oriented helicoids, for instance, the one generated by $\tilde{\Gamma}(s)=(0,0,-s, s)$, contain no singular curves neither singular points.

All these facts obviously hold true for all helicoids with rulings parallel to any contact plane $\Delta_{p}$ and generated by the curve $\Gamma(s)=\left(p+s w, \varphi_{0} \pm s\right)$, where $p=\left(x_{0}, y_{0}, z_{0}\right)$ and $w=\left(\frac{y_{0}}{2},-\frac{x_{0}}{2}, 1\right)$. Indeed, these helicoids can be obtained just by shifting the origin to the point $p$ in the previous example. In particular, it follows that the helicoids of the described class have no singular points. A similar result was also obtained in [6].

Example 3.4. Consider the curve $\Gamma(s)=\left(0,0, \frac{1}{6} s^{3}, s\right)$ for $s \in[0,2 \pi]$. This curve is the integral curve of the vector field

$$
\xi=\frac{\varphi^{2}}{2} X_{3}+X_{4}
$$

starting at the point $\bar{q}_{0}=(0,0,0,0)$. In particular, for any $s \in[0,2 \pi]$ we have $\xi_{1}(s)=\xi_{2}(s)=0, \xi_{3}(s)=\frac{1}{2} s^{2}$ and $\xi_{4}(s)=1$, so that $D(s)=s^{2}$. Therefore $q_{0}$ is the unique singular point of the resulting minimal surface. There are two pairs of simple singular curves $g^{ \pm}(s)=\left( \pm|s| \cos s, \pm|s| \sin s, \frac{1}{6} s^{3}\right), s \neq 0$, whose concatenations (with the point $q_{0}$ ) are smooth curves. In Figure 2 we show the general look of this surface (Fig. 2a) and the structure of its singular set (Fig. 2b). In worth to notice that for this example the genericity condition (2.21) fails and the singular point $q_{0}$ does not give rise to a self-intersection. We will give a simple criterion for the existence of self-intersections for the minimal surfaces in $\mathbb{H}^{1}$ at the end of this section.

Example 3.5. The surface generated by the curve

$$
\Gamma(s)=\left(\sin s,-\cos s, 1+\frac{s}{2}, s\right),
$$

contains a strongly singular curve, actually is it the curve $\gamma=\pi[\Gamma]$. Indeed, $\Gamma$ is the integral curve of the field $\xi=\cos \varphi X_{1}+\sin \varphi X_{2}+X_{4}$. Notice, that despite $\pi_{*}[\xi]=\eta$ condition (2.20) is still verified thanks to the fact that the forth component of the field $\xi$ is different from zero. One can easily check that $D \equiv 0, t_{*}(s) \equiv 0$, and the strongly singular curve is tangent to the characteristic field at every point (Fig. 3).

As we have already seen, sub-Riemannian minimal surfaces generated by purely vertical lines $\left(x_{0}, y_{0}, z_{0}, s\right)$ have isolated singular points. Indeed, it follows that in this case the resulting minimal surface is formed by a one-parametric family of ellipses $\left(t \cos \varphi+x_{0}, t \sin \varphi+y_{0}, \frac{t}{2}\left(x_{0} \sin \varphi-y_{0} \cos \varphi\right)+z_{0}\right), t \geq 0$, that fills the whole plane $\Delta_{p}, p=\left(x_{0}, y_{0}, z_{0}\right)$. In particular, it follows that in $\mathbb{H}^{1}$ the only sub-Riemannian minimal surfaces having isolated characteristic points are planes. Moreover, they can contain at most one isolated singular point (this facts was first noticed in [7]), since they are formed by characteristics which are straight lines, which all intersect at the singular point.

According to Theorem 2.9, any singular characteristic point of type (2.23) is a starting point of a germ of a curve of self-intersections of Whitney's umbrella type. The next proposition describes all possible selfintersections for minimal surfaces in $\mathbb{H}^{1}$ case. This result is an immediate consequence of the explicit parameterization (2.15). Here we denote $v(s)=\eta(\gamma(s)) \in \mathbb{R}^{3}$. 
a)

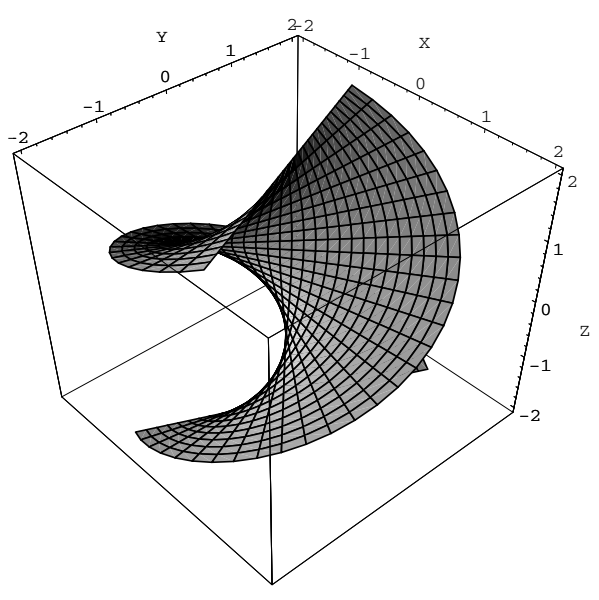

b)

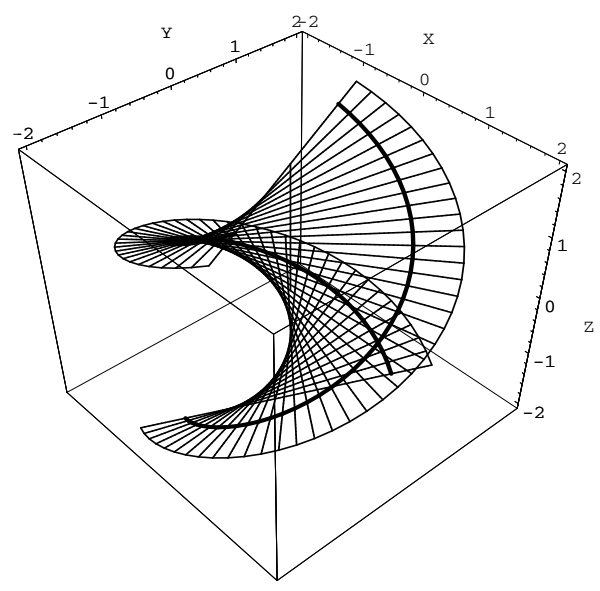

Figure 1. Counter-clockwise oriented helicoid $(t \cos s, t \sin s, s)$ and its singular set.

a)

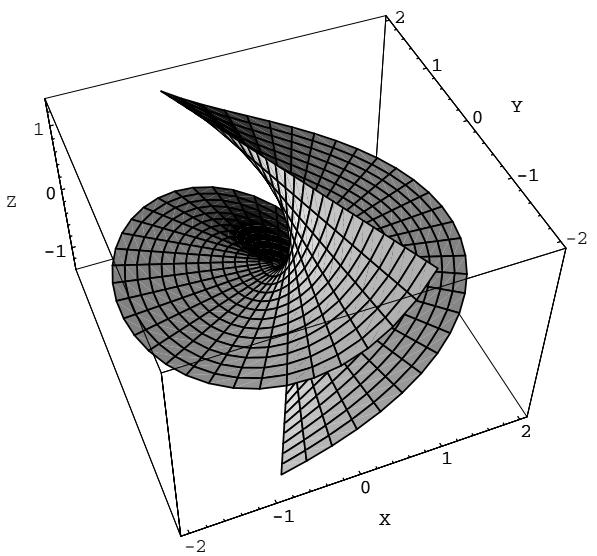

b)

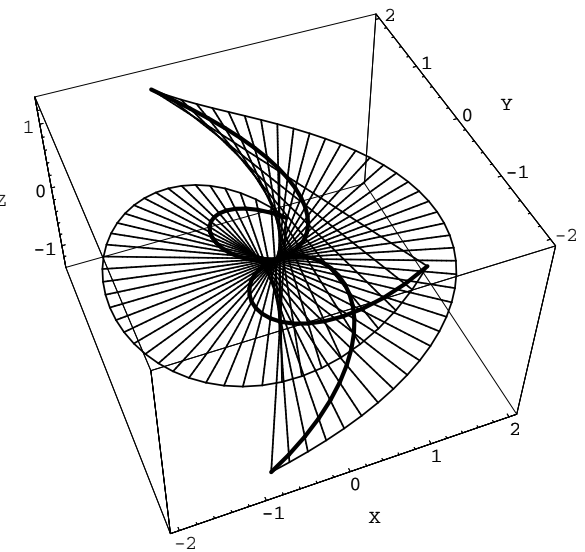

Figure 2. An example of a surface with a pair of simple singular curves and one singular point.

Proposition 3.6. Let $W=\left\{q(t, s): s \in\left[-\delta_{1}, \delta_{2}\right], t \in \mathbb{R}\right\}$ be a piece of sub-Riemannian minimal surface in $\mathbb{H}^{1}$ corresponding to the generating curve $\Gamma(s)=(\gamma(s), \varphi(s))$. If there exist a pair $a, b \in\left[-\delta_{1}, \delta_{2}\right]$ such that $a \neq b$ and a pair of numbers $\tau_{1}, \tau_{2} \in \mathbb{R}$ such that

$$
\gamma(a)-\gamma(b)=\tau_{1} v(a)+\tau_{2} v(b)
$$

then $W$ contains a point of self-intersection $q_{*}=q\left(a,-\tau_{1}\right)=q\left(b, \tau_{2}\right)$.

Example 3.7. Let $\Gamma(s)=(-2 \cos s,-2 \sin s, \cos s, s)$ be the generating curve and denote $\gamma=\pi[\Gamma]$. We have $\xi_{1}(s)=2 \sin s, \xi_{2}(s)=-2 \cos s, \xi_{3}(s)=-2-\sin s$ and $\xi_{4}(s)=1$. First we find the singular points. In the present case $D(s)=-2 \sin s$, i.e., according to Proposition 3.1, the sub-Riemannian minimal surface generated by $\Gamma$ contains two singular points $q(2,0)$ and $q(2, \pi)$. At these points two simple singular curves $q\left(t^{ \pm}(s), s\right)$ branch out. Here $t^{ \pm}(s)=2 \pm \sqrt{-2 \sin s}$ for $s \in(\pi, 2 \pi)$. The concatenation of these simple singular curves (with singular 
a)

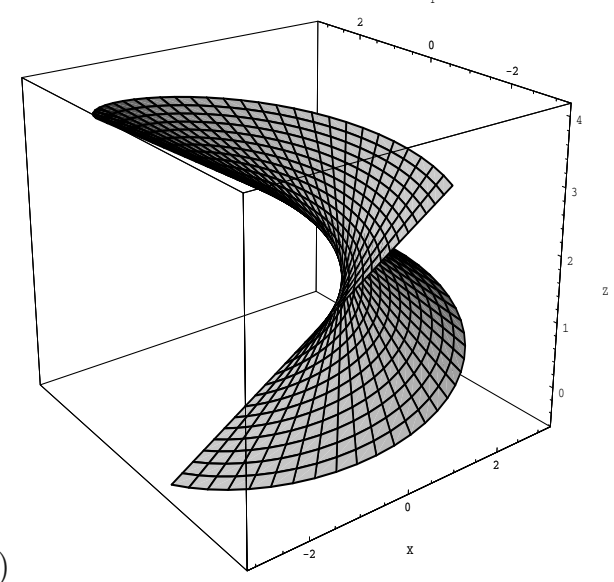

b)

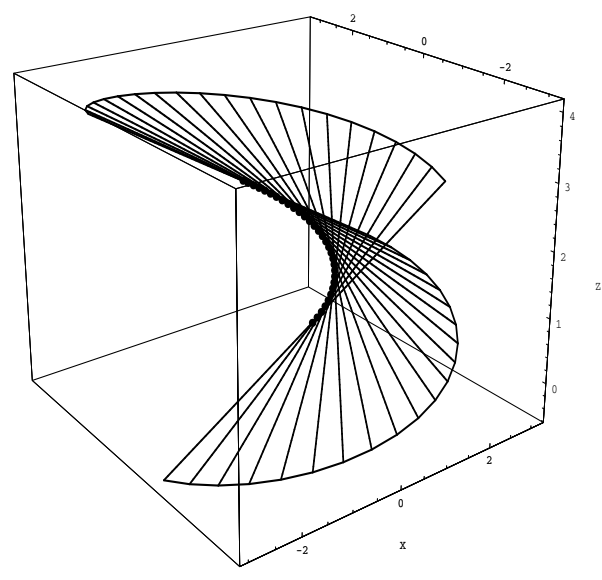

FiguRE 3. A piece of a surface containing a strongly singular curve.

a)

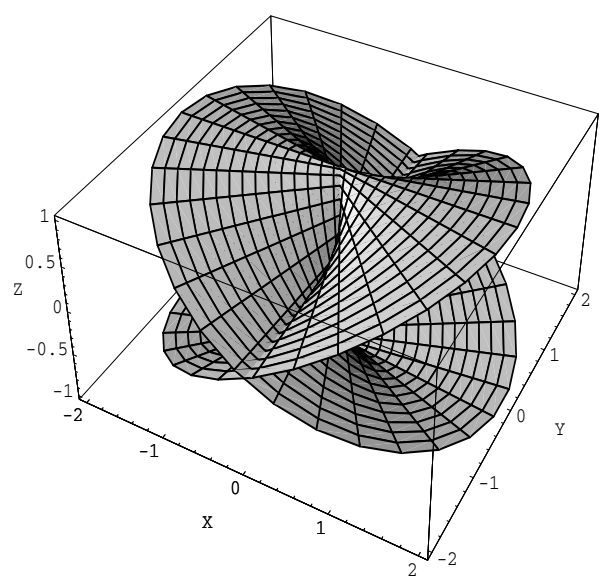

b)

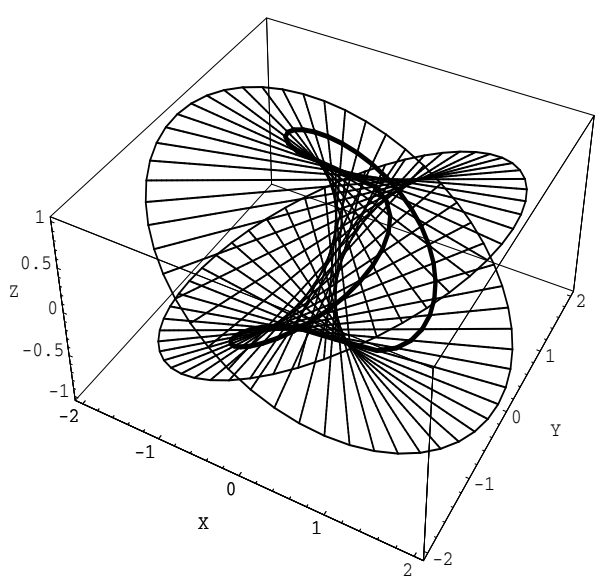

Figure 4. Illustration to Example 3.7.

points) is a unique closed smooth curve. Moreover, at both singular points the genericity assumption (2.21) is verified, so at these points we expect to have two germs of self-intersections.

In order to complete the picture we apply the criterion of Proposition 3.6. Assume that $a$ and $b$ are two distinct numbers on $[0,2 \pi]$. Notice that $v(s)=(\cos s, \sin s, 0)$ for all $s$. Therefore the pairs $a$ and $b$, which may generate self-intersections, necessarily satisfy the relation $\cos a-\cos b=0$. The non-trivial pairs of solutions of this equation are given by the pairs $(a, b)$ where $a=-b \bmod 2 \pi$. Substituting this condition into (3.8) and performing all necessary simplifications we find the following non-trivial pairs of solutions:

$$
a=-b \bmod 2 \pi, \quad \tau_{1}=-2, \tau_{2}=2
$$

Thus the surface in question intersects itself along the segment connecting the singular points $q(2,0)$ and $q(2, \pi)$. Moreover, for $a=\frac{\pi}{2}$ and $b=\frac{3 \pi}{2}$ any pair of numbers $\tau_{i}$ satisfying $\tau_{1}-\tau_{2}+4=0$ is a solution of equation (3.8). 
Therefore the whole line passing throw the points $\gamma\left(\frac{\pi}{2}\right)=(0,-2,0)$ and $\gamma\left(\frac{3 \pi}{2}\right)=(0,2,0)$ is a line of selfintersection. In Figure 4a we show how the sub-Riemannian minimal surface described in this example looks like, and in Figure 4b its singular set (bold curves).

Acknowledgements. The author is grateful to Prof. A. Agrachev whose vision of the problem inspired this work.

\section{REFERENCES}

[1] A.A. Agrachev, Exponential mappings for contact sub-Riemannian structures. J. Dyn. Contr. Syst. 2 (1996) 321-358.

[2] A.A. Agrachev and Yu.L. Sachkov, Control Theory from the Geometric Viewpoint. Berlin, Springer-Verlag (2004).

[3] V.I. Arnold, Geometric Methods in the Theory of Ordinary Differential Equations. Berlin, Springer-Verlag (1988).

[4] V.I. Arnold, Ordinary differential equations. Berlin, Springer-Verlag (1992).

[5] A. Bellä̈che, The tangent space in sub-Riemannian geometry. Progress in Mathematics 144 (1996) 1-78.

[6] J.-H. Cheng and J.-F. Hwang, Properly embedded and immersed minimal surfaces in the Heisenberg group. Bull. Austral. Math. Soc. 70 (2004) 507-520.

[7] J.-H. Cheng, J.-F. Hwang, A. Malchiodi and P. Yang, Minimal surfaces in pseudohermitian geometry. Ann. Scuola Norm. Sup. Pisa Cl. Sci. (5) 4 (2005) 129-177.

[8] J.-H. Cheng, J.-F. Hwang and P. Yang, Existence and uniqueness for p-area minimizers in the Heisenberg group. Math. Ann. 337 (2007) 253-293.

[9] G. Citti and A. Sarti, A cortical based model of perceptual completion in the roto-translation space. J. Math. Imaging Vision 24 (2006) 307-326.

[10] B. Franchi, R. Serapioni and F. Serra Cassano, Rectifiability and perimeter in the Heisenberg group. Math. Ann. 321 (2001) $479-531$.

[11] N. Garofalo and D.-M. Nhieu, Isoperimetric and Sobolev inequalities for Carnot-Carathéodory spaces and the existence of minimal surfaces. Comm. Pure Appl. Math. 49 (1996) 479-531.

[12] N. Garofalo and S. Pauls, The Bernstein problem in the Heisenberg group. Preprint (2004) arXiv:math/0209065v2.

[13] R. Hladky and S. Pauls, Minimal surfaces in the roto-translational group with applications to a neuro-biological image completion model. Preprint (2005) arXiv:math/0509636v1.

[14] R. Montgomery, A tour of subriemannian geometries, their geodesics and applications. Providence, R.I. American Mathematical Society (2002).

[15] S. Pauls, Minimal surfaces in the Heisenberg group. Geom. Dedicata 104 (2004) 201-231.

[16] M. Ritoré and C. Rosales, Rotationally invariant hypersurfaces with constant mean curvature in the Heisenberg group $\mathbb{H}^{n}$. J. Geom. Anal. 16 (2006) 703-720.

[17] H. Whitney, The general type of singularity of a set of $2 n-1$ smooth functions of $n$ variables. Duke Math. J. 10 (1943) 161-172. 